\title{
Theoretical Evaluation of the Hydrodynamic Characteristics of Arrays of Vertical Axisymmetric Floaters of Arbitrary Shape in front of a Vertical Breakwater
}

\author{
Dimitrios N. Konispoliatis ${ }^{1, * \mathbb{D}}$, Spyridon A. Mavrakos ${ }^{1,2}$ and Georgios M. Katsaounis ${ }^{1}$ \\ 1 Laboratory for Floating Structures and Mooring Systems, Division of Marine Structures, School of Naval \\ Architecture and Marine Engineering, National Technical University of Athens, 9 Heroon Polytechniou \\ Avenue, GR 157-73 Athens, Greece; mavrakos@naval.ntua.gr (S.A.M.); katsage@mail.ntua.gr (G.M.K.) \\ 2 Hellenic Centre for Marine Research, Director and President, 19013 Anavyssos, Greece \\ * Correspondence: dkonisp@naval.ntua.gr
}

Received: 12 December 2019; Accepted: 16 January 2020; Published: 20 January 2020

\begin{abstract}
The present paper deals with the analytical evaluation of the hydrodynamic characteristics of an array of vertical axisymmetric bodies of arbitrary shape, placed in front of a reflecting vertical breakwater, which can be conceived as floaters for wave power absorption. At the first part of the paper, the hydrodynamic interactions between the floaters and the adjacent breakwater are exactly taken into account using the method of images, whereas, the interaction phenomena between the floaters of the array are estimated using the multiple scattering approach. For the solution of the problem, the flow field around each floater of the array is subdivided into ring-shaped fluid regions, in each of which axisymmetric eigenfunction expansions for the velocity potential are made. In the second part of the paper, extensive theoretical results are presented concerning the exciting wave forces and the hydrodynamic coefficients for various arrays' arrangements of axisymmetric floaters. The aim of the study is to show parametrically the effect that the vertical breakwater has on the hydrodynamic characteristics of each particular floater.
\end{abstract}

Keywords: vertical axisymmetric floaters; arbitrary shape; breakwater; diffraction and radiation problem; hydrodynamic characteristics; added mass; damping coefficient

\section{Introduction}

Within the context of the linearized theory of water waves, a variety of methods have been devised for the calculation of hydrodynamic interaction phenomena within arrays of floating axisymmetric bodies having vertical symmetry axis. These methods have found application in many areas including the dynamics of oil \& gas offshore platforms, the design of floating airports and other maritime structures. The application of most concern in the present paper is the use of arrays of vertical axisymmetric floaters in front of a vertical breakwater for absorbing wave power.

Several hundreds of patents related to harvesting of wave energy have been in existence by the late 20th century [1-4]. However, despite the increase of interest and awareness for wave energy absorption, the development from concept to commercial stage has been found to be a difficult, slow and expensive process. The main obstacle in harvesting the wave power is the high energy cost, related mainly to the survivability of the wave energy converter (WEC) and its critical components and sub-components (i.e., power take off system, mooring system, power electronics gearbox, etc.,) at the demanding offshore environmental conditions (i.e., extreme weather conditions, salt environment, etc.) [5]. Another aspect for the high energy cost is the often lack of development of an offshore 
grid infrastructure to transport the electricity from renewable offshore energy sources to centers of consumption and storage [6]. Furthermore, the uncertainties in identifying and mitigating the environmental impact of the WEC's life-cycle operation along with the lack of current licensing and consenting procedure lead the developers to face stringent and costly monitoring requirements before and after consent, increasing the wave energy cost [7].

Aiming at overcoming the aforementioned bottlenecks several parameters related to the WEC characteristics have been up to date examined. Representative examples are: (a) the WEC geometrical characteristics optimization, in the scope of harnessing maximum wave energy at the installation location; (b) the optimization of the WEC's characteristics with respect to their mechanical components, to withstand the demanding environmental conditions as well as to reduce the energy losses associated with the transformation of the wave power into electricity; and (c) the installation of WECs close to other near- or on-shore maritime structures such as a breakwater; a harbor or a pier, so as to use the already developed electric grid, reducing in parallel the environmental impact of the WEC's operation [8].

Looking towards the possible advantages provided by installing of WEC devices in near- or on-shore areas or close to other maritime structures, several studies have been presented in the literature. Indicatively, in [9] the most representative existent wave energy converters were evaluated in various offshore and near-shore areas, whereas, in [10] the installation of a WEC device in a port with dual operation, i.e., both as a breakwater as well as a wave energy device was examined.

Furthermore, the effect of a reflecting vertical wall on the WEC 's behavior has been investigated in several studies in the last years. The majority of them are dealing with WECs installed either at a certain distance from the wall or integrated at it. More specifically, in [11] the performance characteristics of an array of five wave energy heaving converters placed in front of a reflecting vertical breakwater have been numerically studied whereas, in [12] the performance of an array of heaving WECs, coupled with DC generators, in front of a breakwater, were numerically and experimentally investigated. Furthermore, solution methods concerning the wave diffraction and radiation problems for the case of a truncated cylinder, in front of a vertical wall have been presented in the literature [13-16], whereas, in [17] the efficiency of a heaving point absorber in front of a vertical wall in regular and irregular seas was studied, based on different floater geometries and wave heading angles. In [18] the possibility of using oscillating water column (OWC) converters for reducing the wave reflection from vertical breakwaters was explored.

As far as investigations concerning the behavior of WECs integrated at a breakwater, theoretical hydrodynamic studies of an oscillating water column device (OWC) placed at the tip of a breakwater and along a straight coast were developed in $[19,20]$. Moreover, numerical analysis and experimental investigation into OWCs integrated at a flat breakwater have been presented in [21] whereas, in [22,23] a feasibility study of an OWC device integrated into a port in the Mediterranean Sea was presented. In $[24,25]$ multiple OWCs structures integrated into floating breakwaters were investigated. Recently, in [26] an analytical study of a pile supported OWC breakwater was presented, whereas, in $[27,28]$ the performance of a WEC integrated into a breakwater was investigated, analytically and experimentally.

Despite the difficulties associated with the placement of WECs in front of a breakwater in terms of installation, mooring and maintenance issues, such converters appear to outperform the integrated into the breakwater counterparts. Their main advantage is the possibility they offer to have them installed at different distances from the vertical wall in dependence from the prevailing sea conditions at the particular installation site, thus optimizing their performance taking advantage of the particular, site-dependent interaction phenomena between the floaters and the breakwater. In addition, WECs placed in front of a breakwater can act as a protecting mole to the incoming waves, reducing the intensity of wave action on the shore at severe environment conditions [8].

As a common wave energy device, an oscillating buoy has been proved to be an effective WEC for wave energy extraction due to its favorable properties: ease of installation, economic operation and manufacturing processes [29-32]. The converter is composed by a base moored to the sea bottom 
and an arbitrary shape floater. The relative motion of the buoyant top to the base is converted into electrical power by the power-take-off (PTO) mechanism. The PTO system can be a high-pressure oil system, converting the floater's motions into hydraulic energy and then converted into electricity through hydraulic motors, or a linear electrical generator [33,34].

The main objective of the present paper is to evaluate the hydrodynamic characteristics (exciting wave forces, hydrodynamic coefficients) of an array of vertical axisymmetric floaters of arbitrary shape that are floating in finite depth waters in front of a vertical and fully reflecting breakwater of infinite length. Towards this goal, the breakwater's effect on the floaters' hydrodynamic coefficients (i.e., hydrodynamic added mass and damping coefficients) and on their exciting wave loads is derived by accounting for the hydrodynamic interaction phenomena both between the bodies of the array as well as between them and the adjacent breakwater.

As far as the simulation of the vertical breakwater is concerned, the method of images is used to describe the fluid flow around the array in front of it. According to this method, the problem of $N$ number of floaters in front of the vertical breakwater is equivalent to the one of an array of $2 \mathrm{~N}$ number of floaters consisting of the initial ones and their image virtual devices with respect to the breakwater that are exposed to the action of surface waves without, however, the presence of the breakwater. The method of images has been initially applied to tackle the diffraction and radiation problems of single or array of cylinders in channels, simulating accurately the reflections from the side walls [35-38]. Later, this method has been also used to simulate the effect of a vertical wall on an array of floating bodies placed in front of it $[11,15,16]$.

Furthermore, considering the solution of the relevant linearized diffraction and radiation problems, the hydrodynamic interference effects between the floaters in the array are evaluated within the context of potential flow theory using single-body hydrodynamic characteristics of the individual bodies and the method of multiple scattering [39-42]. In this formulation, the incident wave potential and various orders of propagating and evanescent wave modes radiated and scattered from all the bodies in the array are superposed to obtain exact series representations of the total wave field around each body of the configuration. As the boundary conditions are satisfied successively on each body in the array, there is no need to retain simultaneously the unknown partial wave amplitudes around all the floaters. As a result, a considerable reduction of the storage requirements in computer applications can be obtained, without, however, compromising the accuracy of the outcomes of the multiple scattering approach compared to other computational methods [43,44].

Finally, in the context of the present contribution, extensive theoretical results concerning the hydrodynamic characteristics (exciting wave forces, hydrodynamic mass and damping coefficients) for several shapes of the individual floaters and array configurations, are given in form of figures and in tabular form. The presented results show that the hydrodynamic characteristics of an array of vertical axisymmetric floaters in front of a vertical wall are evidently different from those in unbounded waters (i.e., without the presence of the breakwater) and their values are dependent form the wave number (i.e., wave frequency), floaters' geometry and arrays' configuration.

\section{Hydrodynamic Formulation}

An array of $N$ vertical axisymmetric floaters, of arbitrary shape, placed in front of a breakwater at constant water depth $d$ is considered. The floaters are exposed to the action of a plane incident wave train of frequency $\omega$ and amplitude $A$ propagating at an angle $\theta$ with respect to the positive $x$-axis. A global, right-handed Cartesian co-ordinate system O-xyz is introduced with origin O located at the still water plane on the breakwater with its vertical axis $\mathrm{Oz}$ directed upwards. Moreover, $N$ local cylindrical co-ordinate systems $\left(r_{q}, \theta_{q}, z_{q}\right), q=1,2, \ldots, N$, are defined with origins at the intersection $\left(X_{q}, Y_{q}\right)$ of the calm water surface with the vertical axis of symmetry of each body. Three different types of floating bodies are examined, (a) a conical floater; (b) a vertical cylindrical floater; and (c) a semi-spherical floater, as seen in Figure 1. All the examined floaters have an outer diameter of $\mathrm{D}$ and distance from the sea bed $h$, whereas the draught of the cylindrical part of the floater, in cases a and c, 
is denoted by $h_{1}$. The distance between the center of the closest to the wall floater and the breakwater is denoted by $l_{\mathrm{W}}$ (see Figure 1).

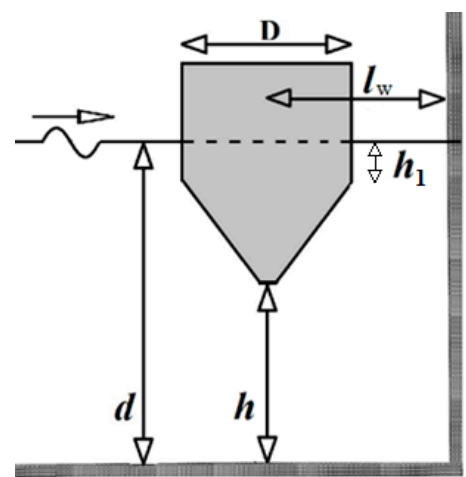

(a)

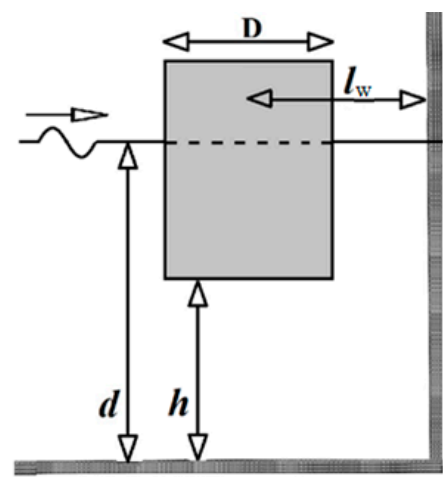

(b)

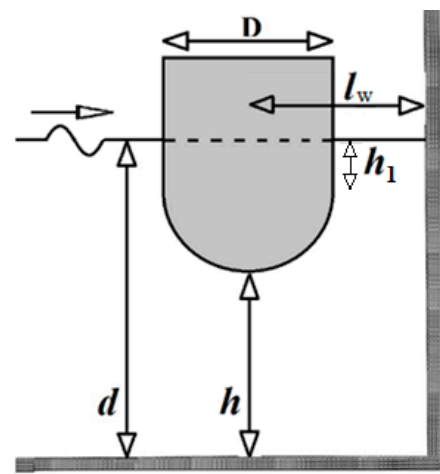

(c)

Figure 1. 2-D representations of the examined types of floaters in front of a breakwater: (a) a conical floater; (b) a vertical cylindrical floater; (c) a semi-spherical floater.

In order to describe the fluid flow around the $N$ floaters of the array in front of the breakwater, the method of images is applied. According to this method, the problem under investigation can be traced back to an equivalent problem of bi-directional incident waves, one propagating at angle $\theta$ and one at angle $180-\theta$ incident on an array of $2 N$ floaters, without the presence of the breakwater (see Figure 2).

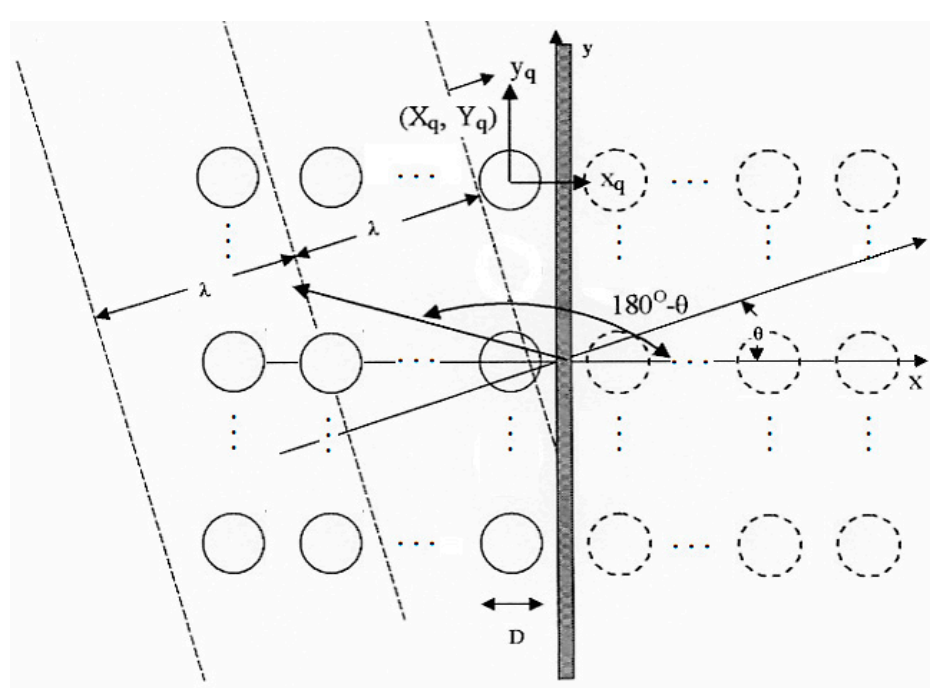

Figure 2. Plan view of the array of $N$ floaters in front of the breakwater (image cylinders are denoted dashed).

Assuming that the flow is irrotational and inviscid and that the waves are of small slope, classical linearized water wave theory can be employed. The fluid flow around each floater $q, q=1$, $\ldots, 2 N$, (including the initial and the image floaters) can be described by the potential function, $\Phi\left(r_{q}, \theta_{q}, z_{q} ; t\right)=\operatorname{Re}\left[\varphi^{q}\left(r_{q}, \theta_{q}, z_{q}\right)\right]$, and expressed, on the basis of linear modeling, as a superposition of incident $\varphi_{0}$, scattered, $\varphi_{s}^{q}$ and radiated wave fields, $\varphi_{j}^{q}$, i.e.,

$$
\varphi^{q}\left(r_{q}, \theta_{q}, z_{q}\right)=\varphi_{0}\left(r_{q}, \theta_{q}, z_{q}\right)+\varphi_{s}^{q}\left(r_{q}, \theta_{q}, z_{q}\right)+\sum_{q=1}^{2 N} \sum_{j=1}^{5} \varphi_{j}^{q}\left(r_{q}, \theta_{q}, z_{q}\right)
$$


Here, $\varphi_{j}^{q}$ denotes the potential of the wave field induced by the forced oscillation of $q$ floater in the $j$-th direction, the remaining ones considered restrained.

The velocity potential of the undisturbed incident wave propagating at an angle $\theta$ with respect to the positive $x$-axis, expressed in the co-ordinate frame of the $q$ floater is:

$$
\varphi_{0}\left(r_{q}, \theta_{q}, z_{q}\right)=-i \omega A \sum_{m=-\infty}^{\infty} i^{m} \Psi_{0, m}\left(r_{q}, z_{q}\right) e^{i m \theta_{q}}
$$

where:

$$
\frac{1}{d} \Psi_{0, m}\left(r_{q}, z_{q}\right)=e^{i k l_{0 q} \cos \left(\theta_{0 q}-\theta\right)} \frac{Z_{0}(z)}{d Z_{0}^{\prime}(0)} J_{m}\left(k r_{q}\right) e^{-i m \theta}
$$

Here, $J_{m}$ is the $m$-th order Bessel function of the first kind; $\left(l_{0 q}, \theta_{0 q}\right)$ are the polar coordinates of the $q$ floater center relative to the origin $\mathrm{O}$ of the global co-ordinate systems $\mathrm{O}-\mathrm{xyz}$ and $\mathrm{Z}_{0}(z)$ are orthonormal functions in $[0, d]$ defined as follows:

$$
Z_{0}(z)=\left[\frac{1}{2}\left[1+\frac{\sinh (2 k d)}{2 k d}\right]\right]^{-1 / 2} \cosh (k(z+d))
$$

In accordance to Equation (2) the diffraction, $\varphi_{D}^{q}=\varphi_{0}+\varphi_{s}^{q}$, and radiation, $\varphi_{j}^{q}$, velocity potentials, around the $q$ floater, when it is considered isolated, can be expressed in the co-ordinate system of body $q$ as follows:

$$
\begin{gathered}
\varphi_{D}^{q}=-i \omega A \sum_{m=-\infty}^{\infty} i^{m} \Psi_{D, m}^{q}\left(r_{q}, z_{q}\right) e^{i m \theta_{q}} \\
\varphi_{j}^{q}=\dot{x}_{j 0}^{q} \sum_{m=-\infty}^{\infty} \Psi_{j, m}^{q}\left(r_{q}, z_{q}\right) e^{i m \theta_{q}} j=1,2, \ldots, 5
\end{gathered}
$$

Here, $\dot{x}_{j 0}^{q}$ is the complex velocity amplitude of $q$ floater's motion in the $j$-th direction.

The velocity potentials, $\varphi_{k}^{q} k=1, \ldots, 5, D$, have to satisfy the Laplace equation within the entire fluid domain; the linearized boundary conditions at the free surface; the zero normal velocity on the sea bed; the kinematic conditions on the mean floater's wetted surface and an appropriate radiation condition at infinity stating that the disturbance propagation must be outgoing [41,42].

The unknown functions $\Psi_{D, m}, \Psi_{j, m}$ involved in Equations (5) and (6) can be established through the method of matched axisymmetric eigenfunctions expansions. According to this method, the flow field around the floater $q$ is subdivided in coaxial ring-shaped fluid regions, denoted by $I$ and $\operatorname{III}_{p}, p=1$, $\ldots, L$ (see Figure 3 ), in which different series expansions of the velocity potential can be established. These series representations are solutions of the Laplace equation and satisfy the kinematic boundary condition at the walls of the floater; the linearized condition at the free surface; the kinematic condition at the sea bed; and the radiation condition at infinity. Moreover, the velocity potentials and their derivatives must be continuous at the vertical boundaries of neighboring fluid regions [45].

By the way of example, the appropriate expansions for the velocity potential, $\Psi_{D, m}, \Psi_{j, m}$, in form of Fourier-Bessel series in the fluid domain $I$ and $I I I_{p}, p=1, \ldots, L$ are presented below.

(a) Infinite ring element $I\left(r \geq \frac{\mathrm{D}}{2}, 0 \leq z \leq d\right)$

$$
\frac{1}{\delta_{k}} \Psi_{k, m}^{q}\left(r_{q}, z\right)=g_{k, m}^{q}\left(r_{q}, z\right)+\sum_{n=0}^{\infty} F_{k, m n}^{q} \frac{K_{m}\left(a_{n} r_{q}\right)}{K_{m}\left(\frac{a_{n} \mathrm{D}}{2}\right)} Z_{n}(z)
$$

for $k=D, 1, \ldots, 5$; 
where:

$$
g_{D, m}^{q}\left(r_{q}, z\right)=\left\{J_{m}\left(k r_{q}\right)-\frac{J_{m}\left(\frac{k \mathrm{D}}{2}\right)}{H_{m}\left(\frac{k \mathrm{D}}{2}\right)} H_{m}\left(k r_{q}\right)\right\} \frac{Z_{0}(z)}{d Z_{0}(d)}
$$

and $\delta_{D}=\delta_{1}=\delta_{2}=\delta_{3}=d, \delta_{4}=\delta_{5}=d^{2} ; H_{m}, K_{m}$ are the $m$-th order Hankel function of first kind and the modified Bessel function of second kind, respectively; $F_{k, m n}^{q}$ are the unknown Fourier coefficients to be determined by the solution procedure. Furthermore,

$$
Z_{n}(z)=\left[\frac{1}{2}\left[1+\frac{\sin \left(2 a_{n} d\right)}{2 a_{n} d}\right]\right]^{-1 / 2} \cos \left(a_{n}(z+d)\right), n \geq 1
$$

The eigenvalues $a_{n}$ are roots of the transcendental equation: $\omega^{2}+g a_{n} \tan \left(a_{n} d\right)=0$, which possesses one imaginary, $a_{0}=-i k, k>0$ and infinite number of real roots.

(b) $p$-th ring element $I I I_{p}$ of the $q$ floater $\left(\alpha_{p} \leq r_{p} \leq \alpha_{p+1}, 0 \leq z \leq h_{p}, p=1,2, \ldots, L\right)$

$$
\frac{1}{\delta_{k}} \Psi_{k, m}^{q}\left(r_{p}, z\right)=g_{k, m}^{q}\left(r_{p}, z\right)+\sum_{n_{p}=0}^{\infty} \epsilon_{n_{p}}\left[R_{m n_{p}}\left(r_{p}\right) F_{k, m n_{p}}^{q}+R_{m n_{p}}^{*}\left(r_{p}\right) F_{k, m n_{p}}^{* q}\right] \cos \left(\frac{n_{p} \pi(z+d)}{h_{p}}\right)
$$

Here: $g_{D, m}^{q}\left(r_{p}, z\right)=g_{1, m}^{q}\left(r_{p}, z\right)=g_{2, m}^{q}\left(r_{p}, z\right)=0 ; g_{3, m}^{q}\left(r_{p}, z\right)=\frac{(z+d)^{2}-\left(\frac{1}{2}\right) r_{p}^{2}}{2 h_{p} d} ; g_{4, m}^{q}\left(r_{p}, z\right)=$ $-g_{5, m}^{q}\left(r_{p}, z\right)=\frac{-r_{p}\left[(z+d)^{2}-\left(\frac{1}{4}\right) r_{p}^{2}\right]}{2 h_{p} d^{2}} ; \delta_{k}$ has been defined above; $F_{k, m n_{p}}^{q}, F_{k, m n_{p}}^{* q}$ are Fourier coefficients to be determined by the solution procedure; $\epsilon_{n_{p}}$ is Neumann's symbol defined as: $\epsilon_{n_{p}}=1$, for $n_{p}=0$; otherwise $\epsilon_{n_{p}}=2$.

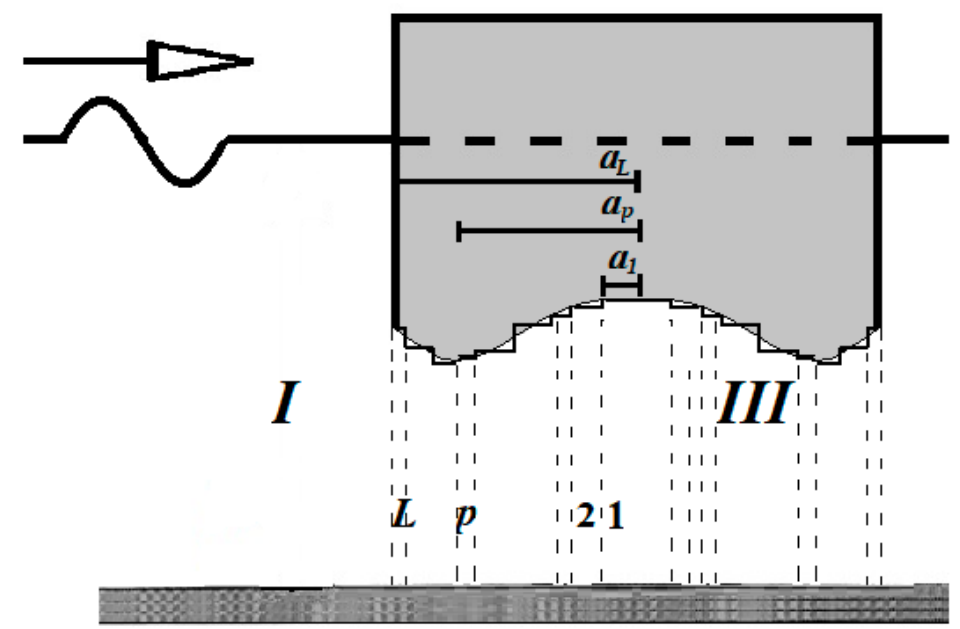

Figure 3. Discretization of the flow field around an axisymmetric arbitrary shape floater.

The $R_{m n_{p}}, R_{m n_{p}}^{*}$ terms express the radial dependence of the fluid's velocity in the $p$-th ring element. They can be written as:

$$
\begin{aligned}
& R_{m 0}\left(r_{p}\right)=\frac{\left(\frac{r_{p}}{\alpha_{p}}\right)^{m}-\left(\frac{\alpha_{p}}{r_{p}}\right)^{m}}{\left(\frac{\alpha_{p+1}}{\alpha_{p}}\right)^{m}-\left(\frac{\alpha_{p}}{\alpha_{p+1}}\right)^{m}}, R_{m 0}^{*}\left(r_{q}\right)=\frac{\left(\frac{\alpha_{p+1}}{r_{p}}\right)^{m}-\left(\frac{r_{p}}{\alpha_{p+1}}\right)^{m}}{\left(\frac{\alpha_{p+1}}{\alpha_{p}}\right)^{m}-\left(\frac{\alpha_{p}}{\alpha_{p+1}}\right)^{m}}, n_{p}=0 \\
& R_{m 0}\left(r_{p}\right)=\frac{K_{m}\left(\frac{n_{p} \pi \alpha_{p}}{h_{p}}\right) I_{m}\left(\frac{n_{p} \pi r_{p}}{h_{p}}\right)-I_{m}\left(\frac{n_{p} \pi \alpha_{p}}{h_{p}}\right) K_{m}\left(\frac{n_{p} \pi r_{p}}{h_{p}}\right)}{K_{m}\left(\frac{n_{p} \pi \alpha_{p}}{h_{p}}\right) I_{m}\left(\frac{n_{p} \pi \alpha_{p+1}}{h_{p}}\right)-I_{m}\left(\frac{n_{p} \partial \alpha_{p}}{h_{p}}\right) K_{m}\left(\frac{n_{p} \pi \alpha_{p+1}}{h_{p}}\right)}, n_{p} \neq 0
\end{aligned}
$$




$$
R_{m 0}^{*}\left(r_{p}\right)=\frac{I_{m}\left(\frac{n_{p} \pi \alpha_{p+1}}{h_{p}}\right) K_{m}\left(\frac{n_{p} \pi r_{p}}{h_{p}}\right)-K_{m}\left(\frac{n_{p} \pi \alpha_{p+1}}{h_{p}}\right) I_{m}\left(\frac{n_{p} \pi r_{p}}{h_{p}}\right)}{K_{m}\left(\frac{n_{p} \pi \alpha_{p}}{h_{p}}\right) I_{m}\left(\frac{n_{p} \pi \alpha_{p+1}}{h_{p}}\right)-I_{m}\left(\frac{n_{p} \pi \alpha_{p}}{h_{p}}\right) K_{m}\left(\frac{n_{p} \pi \alpha_{p+1}}{h_{p}}\right)}, n_{p} \neq 0
$$

Especially, for $p=1$ (the middle fluid region underneath the $q$ floater) the velocity potential is given as:

$$
\frac{1}{\delta_{k}} \Psi_{k, m}^{q}\left(r_{p}, z\right)=g_{k, m}^{q}\left(r_{p}, z\right)+\sum_{n_{1}=0}^{\infty} \epsilon_{n_{1}} F_{k, m n_{1}}^{q} \frac{I_{m}\left(\frac{n_{1} r_{1}}{h}\right)}{I_{m}\left(\frac{n_{1} \pi a_{1}}{h}\right)} \cos \left(\frac{n_{1} \pi(z+d)}{h}\right)
$$

In accordance to the Equations (5) and (6) the diffraction and radiation velocity potential induced around any floater of the array can be written as:

$$
\begin{gathered}
\varphi_{D}^{q q}=-i \omega A \sum_{m=-\infty}^{\infty} i^{m} \Psi_{D, m}^{q q}\left(r_{q}, z_{q}\right) e^{i m \theta_{q}} \\
\varphi_{j}^{q p}=\dot{x}_{j 0}^{p} \sum_{m=-\infty}^{\infty} \Psi_{i, m}^{q p}\left(r_{q}, z_{q}\right) e^{i m \theta_{q}}
\end{gathered}
$$

Here $\Psi_{D, m}^{q q}$, is the diffraction potential due to the interference of the $q$ floater with the incoming incident wave field and the scattered waves by all floaters in the array, whereas $\Psi_{i, m}^{q p}$ denote the radiation potentials around the $q$ floater of the array due to the forced oscillation of the $p$ floater.

In order to express the potentials in form of Equations (15) and (16) the multiple scattering method is applied, taking into consideration the interaction phenomena between the bodies of the array. The method which is applicable to arrays consisting of an arbitrary number of vertical axisymmetric bodies, having any geometrical arrangement and individual body geometry, has been described exhaustively in previous publications [41,42], thus it is not further elaborated here.

\section{Hydrodynamic Reaction Forces}

Having determined the diffraction and radiation velocity potentials around each floater of the array the exciting wave forces and the hydrodynamic reaction forces acting on the $q$ floater; $(q=1,2$, $2 N)$ can be obtained by:

$$
\begin{aligned}
F_{D, i}^{q} & =-\iint_{S_{q}} i \omega \rho \varphi_{D}^{q q} \mathrm{n}_{i} d S \\
F_{i j}^{q p} & =-\iint_{S_{q}} i \omega \rho \varphi_{j}^{q p} \mathrm{n}_{i} d S
\end{aligned}
$$

Here, $F_{D, i}^{q}$ denotes the exciting wave force acting on the $q$ floater in the $i$-th direction; $F_{i j}^{q p}$ are the hydrodynamic reaction forces acting on the $q$ floater in the $i$-th direction, due to the forced oscillation of the $p$ floater in the $j$-th direction; $\rho$ is the water density; $S_{q}$ is the mean wetted surface of the $q$ floater; $\mathrm{n}_{i}$ are the generalized normal components defined by: $\mathrm{n}=\left(\mathrm{n}_{1}, \mathrm{n}_{2}, \mathrm{n}_{3}\right) ; \mathrm{r} \times \mathrm{n}=\left(\mathrm{n}_{4}, \mathrm{n}_{5}\right) ; \mathrm{r}$ being the position vector of a point on the wetted surface $S_{q}$ with respect to the reference co-ordinate system of body $q$.

The hydrodynamic reaction forces $F_{i j}^{q p}$ can be also written as [46]:

$$
F_{i j}^{q p}=i \omega\left(a_{i, j}^{q, p}+\frac{i}{\omega} \beta_{i, j}^{q, p}\right) \dot{x}_{j 0}^{p}
$$

where $a_{i, j}^{q, p}, \beta_{i, j}^{q, p}$ are the added mass and damping coefficients, respectively, of the $q$ floater in $i$-th direction due to the forced oscillation of the $p$ floater in the $j$-th direction.

Based on the method of images the exciting forces acting on the $q$ floater of an array of $N$ floaters in front of a vertical breakwater exposed to the action of waves propagating at an angle $\theta$, equal to the 
sum of the exciting forces acting on the initial $q$ floater, for wave angles $\theta$ and $180-\theta$, assuming the presence of image floaters, with respect to the breakwater (i.e., total number of floaters $2 N$ ), without the presence of the vertical wall. Furthermore, the hydrodynamic coefficients $a_{i, j}^{q, p}, \beta_{i, j}^{q, p}$ of the $q$ floater in $i$-th direction due the forced oscillation of the $p$ floater in $j$-th mode of motion in front of the vertical breakwater can be derived, by summing up properly the motion-dependent hydrodynamic coefficients $a_{i, j}^{q, p}, b_{i, j}^{q, p}$ of the initial $q$ floater in the $i$-th direction (i.e., $\left.I=1, \ldots, 5\right)$ due to the forced oscillation of the $p$ floater in the $j$-th direction (i.e., $j=1, \ldots, 5$ ) with the corresponding hydrodynamic coefficients $a_{i, j}^{q, p^{\prime}}, b_{i, j}^{q, p^{\prime}}$ of the initial $q$ floater due to the forced oscillation in the $j$-th direction of the image floater of the $p$ body, denoted as $p$. Table 1 denotes, indicatively, the determination of the added mass coefficient of the $q$ floater of an array of $N$ floaters in front of a vertical wall, using the image theory. The same formula is applied to the damping coefficients.

Table 1. Added mass coefficients of the $q$ floater, $q=1, \ldots, N$, in front of a vertical wall, using the image theory (the image floater of the $p$ floater is denoted as $p^{\prime}$ ).

\begin{tabular}{rcccc}
\hline$a_{1,1}^{q, p}-a_{1,1}^{q, p^{\prime}}$ & $a_{1,2}^{q, p}+a_{1,2}^{q, p^{\prime}}$ & $a_{1,3}^{q, p}+a_{1,3}^{q, p^{\prime}}$ & $a_{1,4}^{q, p}+a_{1,4}^{q, p^{\prime}}$ & $a_{1,5}^{q, p}-a_{1,5}^{q, p^{\prime}}$ \\
\hline$a_{2,1}^{q, p}-a_{2,1}^{q, p^{\prime}}$ & $a_{2,2}^{q, p}+a_{2,2}^{q, p^{\prime}}$ & $a_{2,3}^{q, p}+a_{2,3}^{q, p p^{\prime}}$ & $a_{2,4}^{q, p}+a_{2,4}^{q, p^{\prime}}$ & $a_{2,5}^{q, p}-a_{2,5}^{q, p^{\prime}}$ \\
\hline$a_{3,1}^{q, p}-a_{3,1}^{q, p^{\prime}}$ & $a_{3,2}^{q, p}+a_{3,2}^{q, p^{\prime}}$ & $a_{3,3}^{q, p}+a_{3,3}^{q, p^{\prime}}$ & $a_{3,4}^{q, p}+a_{3,4}^{q, p^{\prime}}$ & $a_{3,5}^{q, p}-a_{3,5}^{q, p^{\prime}}$ \\
\hline$a_{4,1}^{q, p}-a_{4,1}^{q, p^{\prime}}$ & $a_{4,2}^{q, p}+a_{4,2}^{q, p^{\prime}}$ & $a_{4,3}^{q, p}+a_{4,3}^{q, p^{\prime}}$ & $a_{4,4}^{q, p}+a_{4,4}^{q, p^{\prime}}$ & $a_{4,5}^{q, p}-a_{4,5}^{q, p^{\prime}}$ \\
\hline$a_{5,1}^{q, p}-a_{5,1}^{q, p^{\prime}}$ & $a_{5,2}^{q, p}+a_{5,2}^{q, p^{\prime}}$ & $a_{5,3}^{q, p}+a_{5,3}^{q, p^{\prime}}$ & $a_{5,4}^{q, p}+a_{5,4}^{q, p^{\prime}}$ & $a_{5,5}^{q, p}-a_{5,5}^{q, p^{\prime}}$ \\
\hline
\end{tabular}

It should be also noted that the Table 1 fulfils the symmetry requirements of the added mass coefficients, i.e., $a_{1,3}^{q, p}+a_{1,3}^{q, p^{\prime}}=a_{3,1}^{q, p}-a_{3,1}^{q, p^{\prime}} ; a_{1,5}^{q, p}-a_{1,5}^{q, p^{\prime}}=a_{5,1}^{q, p}-a_{5,1}^{q, p^{\prime}} ; a_{4,2}^{q, p}+a_{4,2}^{q, p^{\prime}}=a_{2,4}^{q, p}+a_{2,4}^{q, p^{\prime}} ;$ etc.

\section{Numerical Results}

Initially, the theoretical results derived from the aforementioned analysis are compared with the available ones from the literature. The present analytical model is applied to the case of a single floating cylinder located in front of a vertical wall at finite water depth in order to compare the results with the ones of $[15,16]$. The examined cylinder of radius $\mathrm{D} / 2$ and draught $2(d-h) / \mathrm{D}=0.5$ is subjected to incident wave with an angle of attack $\theta=0$ (i.e., the wave is propagating along the $x$ axis), at a water depth $2 d / \mathrm{D}=1.0$, for various examined distances between the center of the cylinder and the vertical wall $2 l_{\mathrm{w}} / \mathrm{D}$ (see Figure 1). The comparison is made, indicatively, in terms of the dimensionless surge exciting forces (see Equation (17)), i.e., $F=\frac{\left|F_{D, 1}^{1}\right|}{\frac{\pi \rho g D^{2} A}{4}}$; and the dimensionless hydrodynamic coefficients (see Equation (19)), i.e., $\mu_{i}=\frac{a_{1, i}^{1,1}}{\frac{\pi \rho D^{2}(d-h)}{4}}, i=1,3,5 ; c_{i}=\frac{b_{1, i}^{1,1}}{\frac{\omega \pi \rho D^{2}(d-h)}{4}}, i=1,3,5$.

Figure 4 depicts the surge exciting forces for various examined distances between the device and the wall. An excellent correlation between the analytical results of the presented theoretical method and the analytical results from [15] can be obtained. In the Figure 5 the dimensionless surge hydrodynamic coefficients (i.e., hydrodynamic added mass and damping) of the floating cylinder due to its forced oscillations in surge, heave and pitch directions, are presented and compared, with also an excellent agreement, with the analytical results from [16]. 


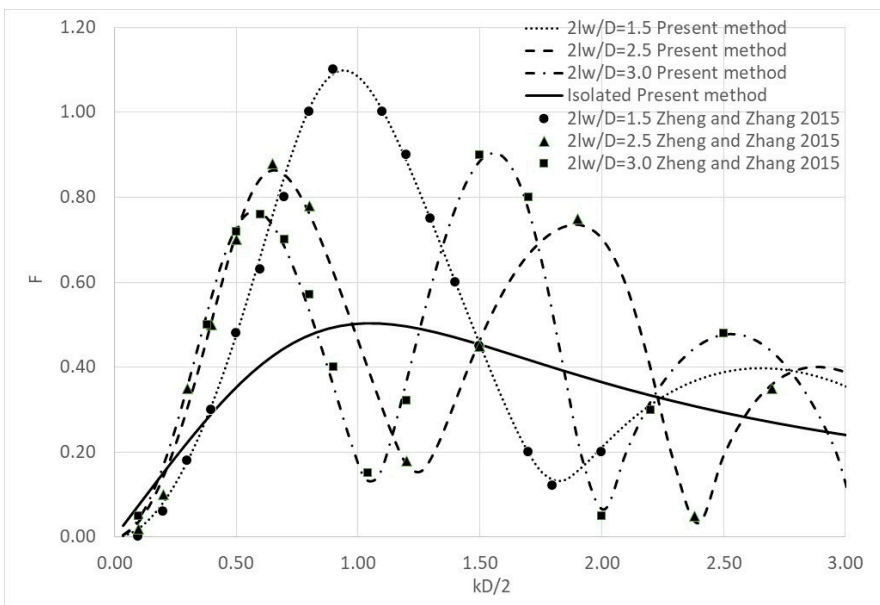

Figure 4. Dimensionless surge wave exciting forces against $k \mathrm{D} / 2$ for three distances between the floater and the vertical wall and for an isolated floater (i.e., without the presence of the breakwater). The results are compared with the analytical results of [15].

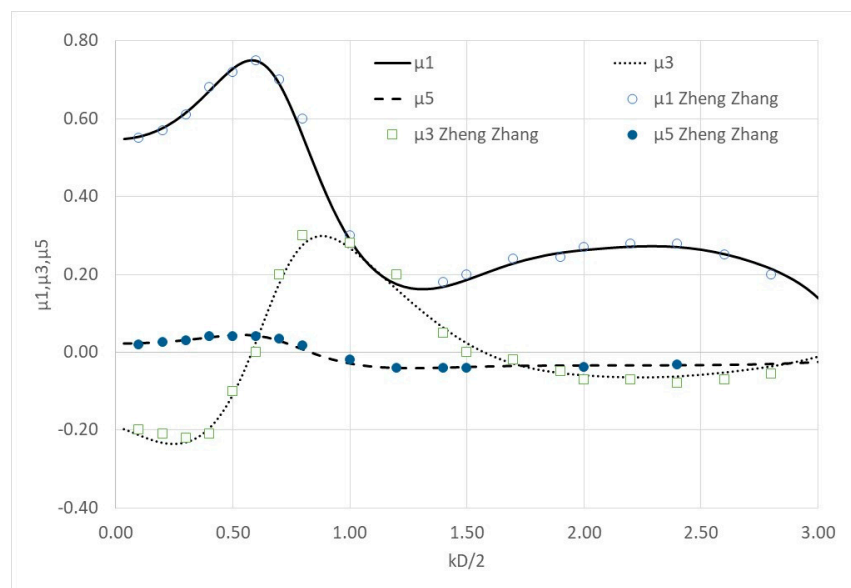

(a)

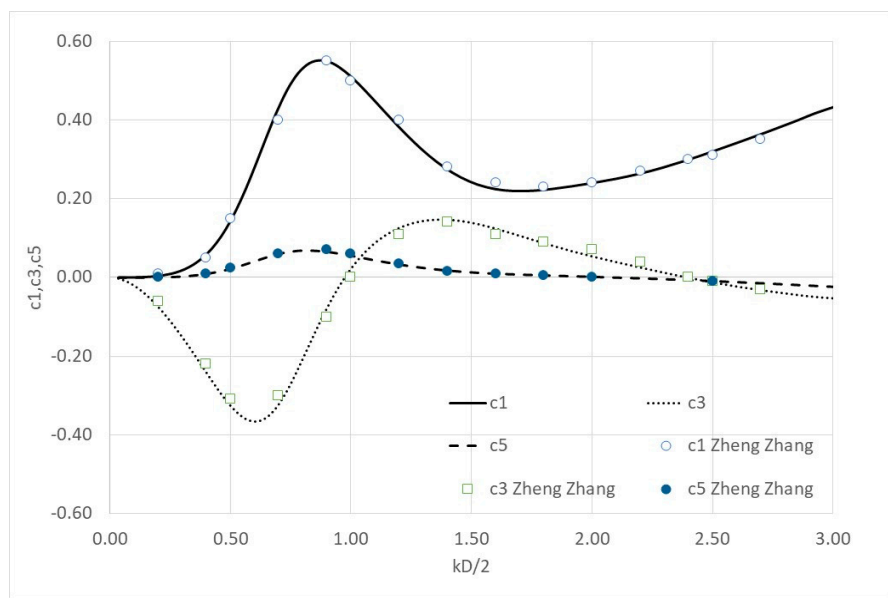

(b)

Figure 5. Dimensionless surge added mass (a) and surge damping coefficients (b) against $k \mathrm{D} / 2$ due to motions of the cylindrical body in surge, heave and pitch, for $l_{\mathrm{w}} / \mathrm{D}=1$. The results are compared with the analytical ones of [16]. 
Next, three different array types of axisymmetric floaters with vertical symmetry axes are examined, (a) an array of five same conical floaters; (b) an array of five same vertical cylindrical floaters; and (c) an array of five same semi-spherical floaters (see Figure 1). The floaters are placed in front of a vertical breakwater of infinite length in three different array configurations, i.e., the devices are placed: (a) in a parallel direction to the wall; (b) in a rectangular arrangement in front of the wall; and (c) in a perpendicular direction to the wall; see Figure $6, C_{1}, C_{2}, C_{3}$, respectively. The wave is assumed to propagate along the $x$-axis. The distance between the center of the closest to the wall floater and the breakwater is $l_{\mathrm{W}}$, whereas the distance between adjacent bodies is $l_{\mathrm{b}}$ (in $C_{2}$ array-see Figure 6 - the distance of the 4 th from the 5 th device and of the 1 st from the 2 nd device is $1.732 l_{\mathrm{b}}$ ). The examined floaters have a radius: $\mathrm{D} / 2$; draught: $(d-h) / \mathrm{D}=0.5$; and $\mathrm{D} /\left(2 h_{1}\right)=10$; at a water depth: $d / \mathrm{D}=1.0$ (see Figure 1). The distances between the center of the cylinder and the vertical wall and between the adjacent cylinders are $l_{\mathrm{w}} / \mathrm{D}=2$ and $l_{\mathrm{b}} / \mathrm{D}=4$, respectively.

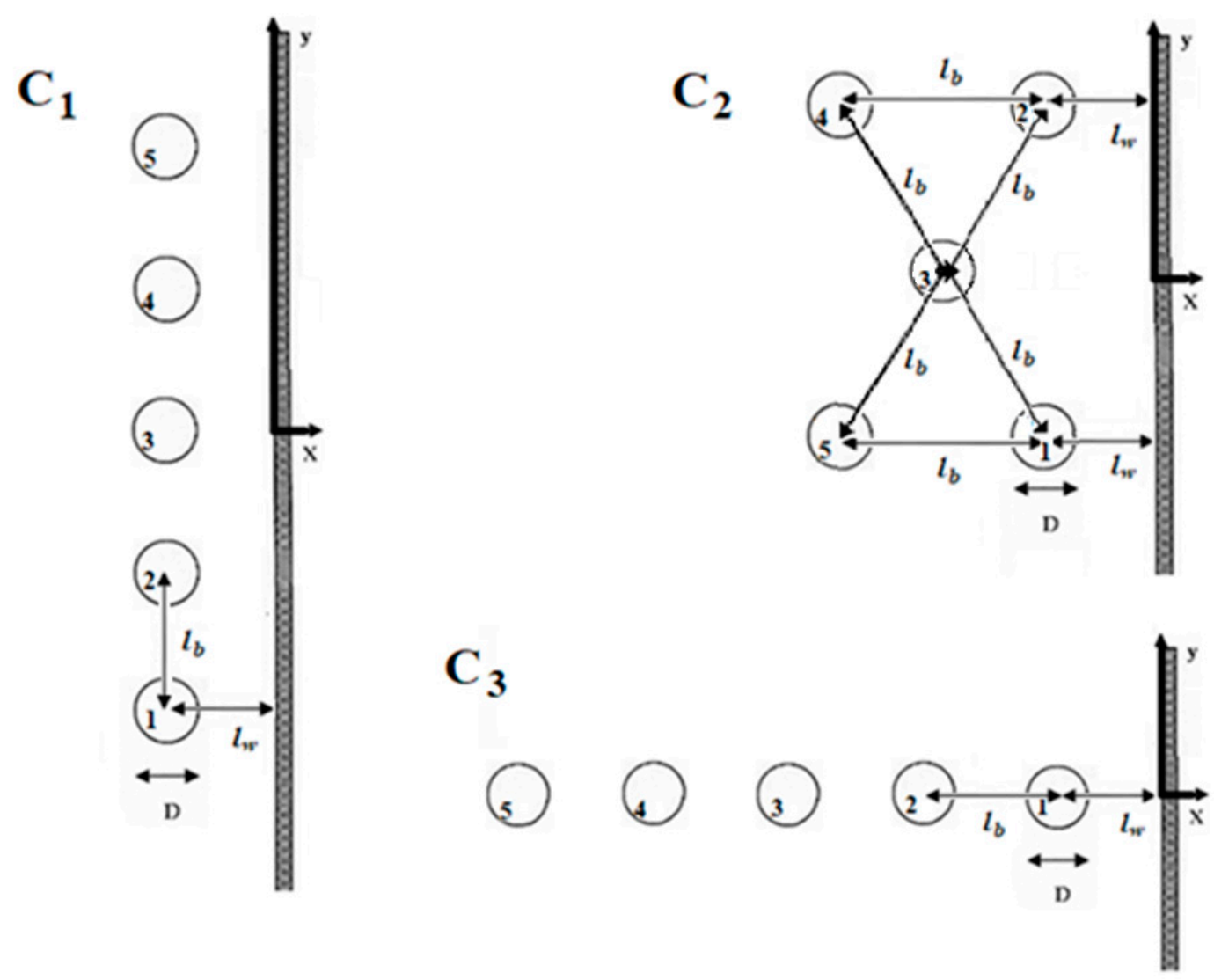

Figure 6. Three different examined array configurations concerning the position of each floater to the incoming wave and the vertical breakwater. The array $C_{1}$ is placed in a parallel direction to the wall; the array $C_{2}$ in a rectangular arrangement in front of the wall; and the array $C_{3}$ is placed in a perpendicular direction to the wall.

In the Figures 7 and 8 the dimensionless wave exciting forces in surge (see Equation (17)), i.e., $\mathrm{F}_{x}=\frac{\left|F_{D, 1}^{k}\right|}{\frac{\pi \rho g D^{2} A}{4}}$; acting on the $k$ floater, i.e., $k=1,3$ of each examined array configuration in front of a breakwater (see Figure 6), are plotted against the corresponding values acting on the same floater of the array without, however, the presence of the vertical wall (i.e., no-wall cases in the figures) versus $\mathrm{kD} / 2$. Here $k$ denotes the wave number and $\mathrm{D}$ the diameter of the converter. Also, the Figures 9 and 10 depict the vertical counterpart of the dimensionless exciting forces i.e., $\mathrm{F}_{z}=\frac{\left|F_{D, 3}^{k}\right|}{\frac{\pi \rho \mathrm{D}^{2} A}{4}}$; acting on the $k$ 
floater, i.e., $k=1,3$ compared also with the heave wave loads on the same floater of the array, without the presence of the breakwater (i.e., no-wall cases in the figures), against $\mathrm{kD} / 2$.

It can be seen from the Figure 7 that due to the reflected waves from the breakwater the values of the surge exciting forces oscillate around the corresponding values of the same device of the array, without the presence of the wall. Furthermore, it is also evident in the Figure 7 that the horizontal exciting forces are minimizing at $\mathrm{kD} / 2=0.80 ; 1.57 ; 2.36 ; \ldots$ etc. regardless the shape of the floater or the array configuration. However, this is not the case for the same examined floater and array configuration without the presence of the vertical wall. This phenomenon can be traced back to the interaction effects between the breakwater and the floaters. More specifically, each of these wave numbers (i.e., wave frequencies) correspond to a wavelength equals to a multiple value of the distance between the initial and the image floater, (i.e., $2 l_{\mathrm{w}}$ ).

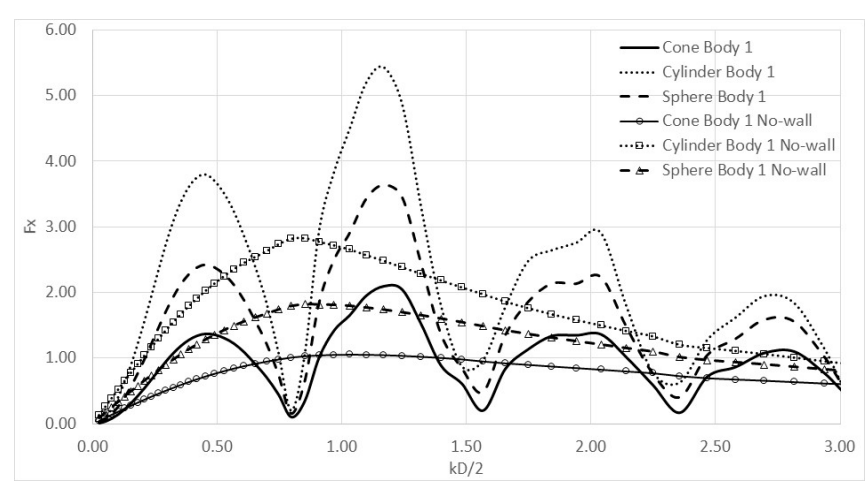

(a)

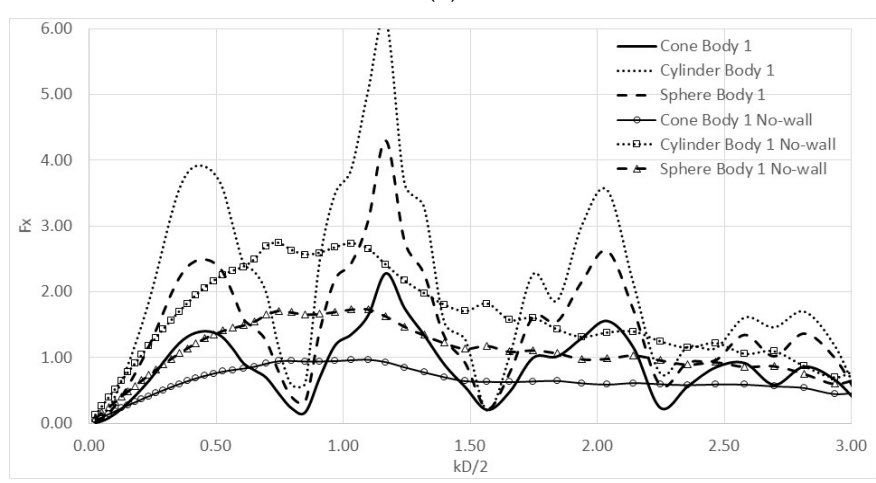

(b)

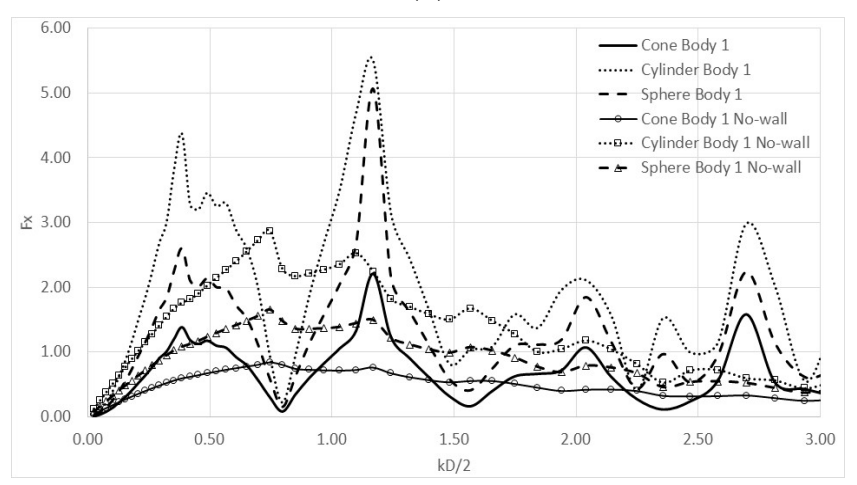

(c)

Figure 7. Dimensionless horizontal exciting wave forces acting on the 1st floater of the arrays against $\mathrm{kD} / 2$, for the three different examined array configurations. Comparison with the surge exciting wave forces on the same floater of the arrays without the presence of the breakwater: (a) parallel arrangement, $\mathrm{C}_{1} ;(\mathbf{b})$ rectangular arrangement, $\mathrm{C}_{2} ;(\mathbf{c})$ perpendicular arrangement, $\mathrm{C}_{3}$. 
Moreover, it is also depicted that the horizontal exciting forces on the 1st cylinder of the cylindrical floater array are larger comparing with those on the conical and the semi-spherical floater-array. The reason is the volume of the cylindrical floater which is larger than the volume of the semi-spherical and the conical floater. Finally, it can be seen from the Figure $7 \mathrm{~b}, \mathrm{c}$, that the scattered waves between the remaining bodies of the perpendicular and rectangular arrangement and the examined floater, create additional peaks (i.e., at $\mathrm{kD} / 2 \approx 0.6 ; 1.7 ; 2.4$ ) on the horizontal forces compared with the corresponding values on the 1st floater of the parallel arrangement. This can be traced back to the position of the floaters of each configuration with respect to the incoming wave train.

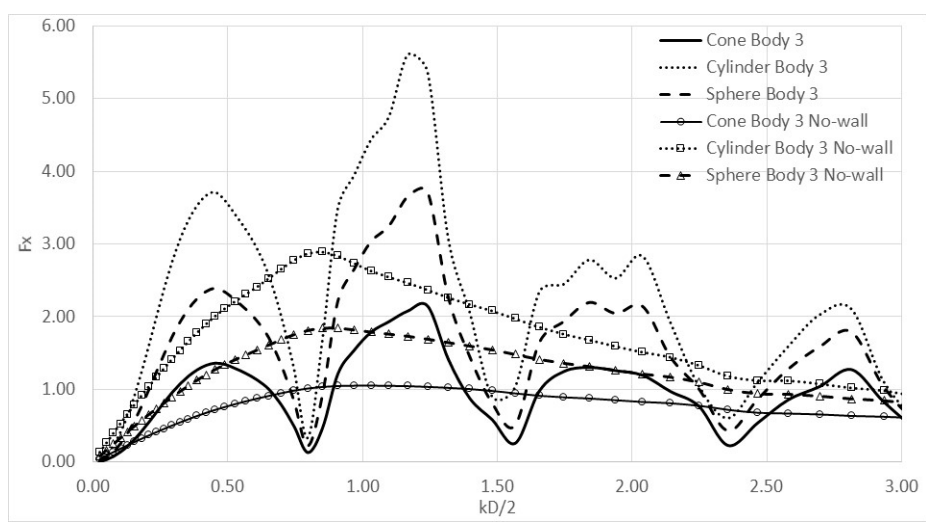

(a)

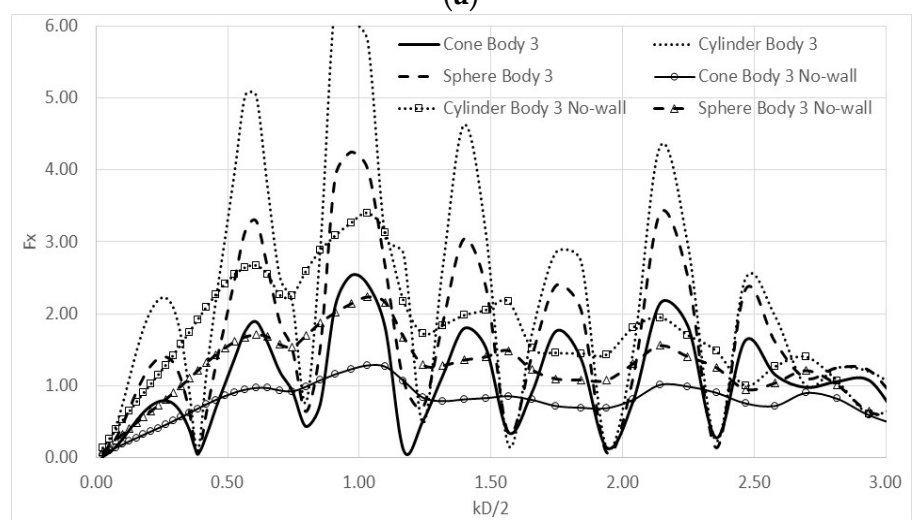

(b)

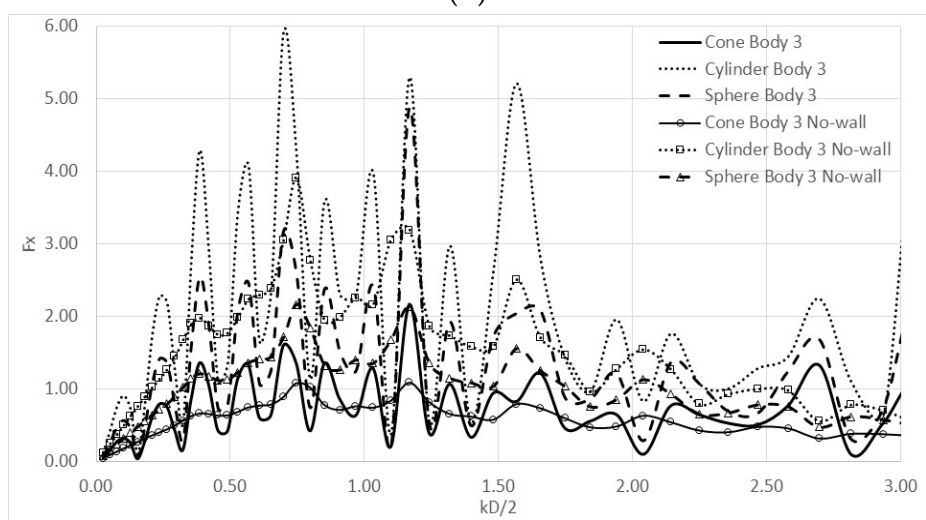

(c)

Figure 8. Dimensionless horizontal exciting wave forces acting on the 3rd floater of the arrays plotted against the non-dimensional wave number $\mathrm{kD} / 2$, for the three different examined array configurations. Comparison with the surge exciting wave forces on the same floater of the arrays without the presence of the breakwater: (a) parallel arrangement, $\mathrm{C}_{1} ;(\mathbf{b})$ rectangular arrangement, $\mathrm{C}_{2} ;$ (c) perpendicular arrangement, $\mathrm{C}_{3}$. 


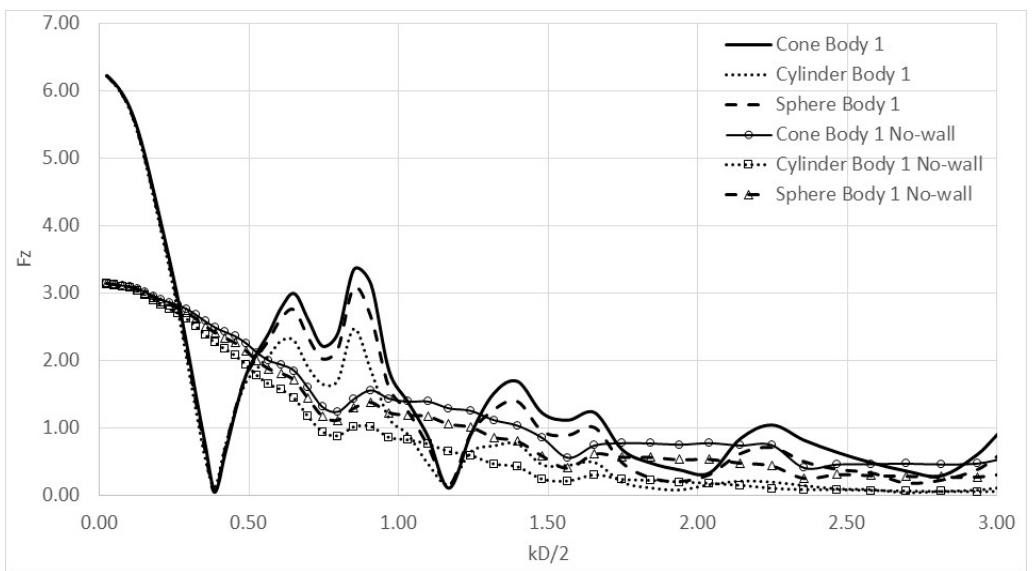

(a)

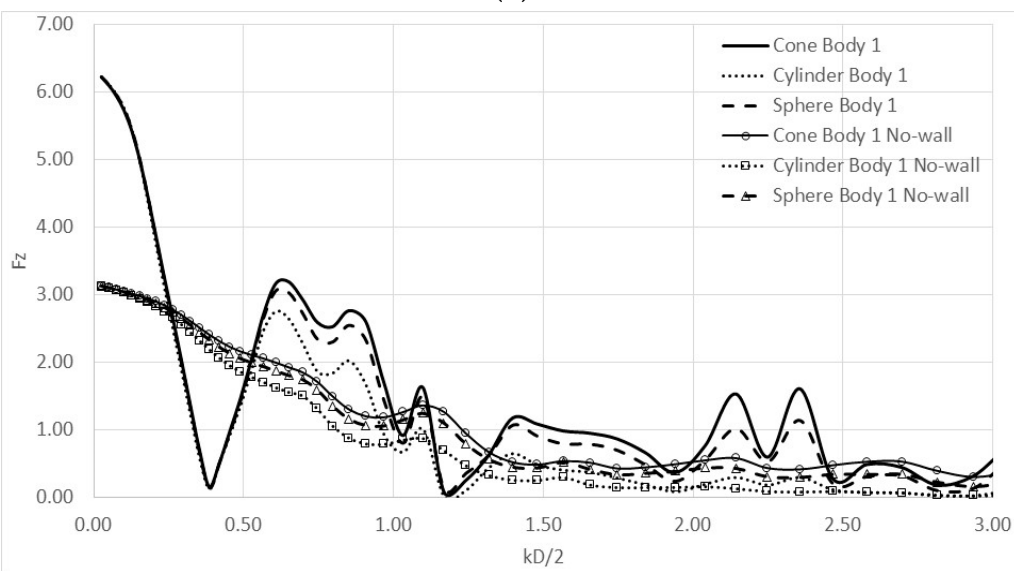

(b)

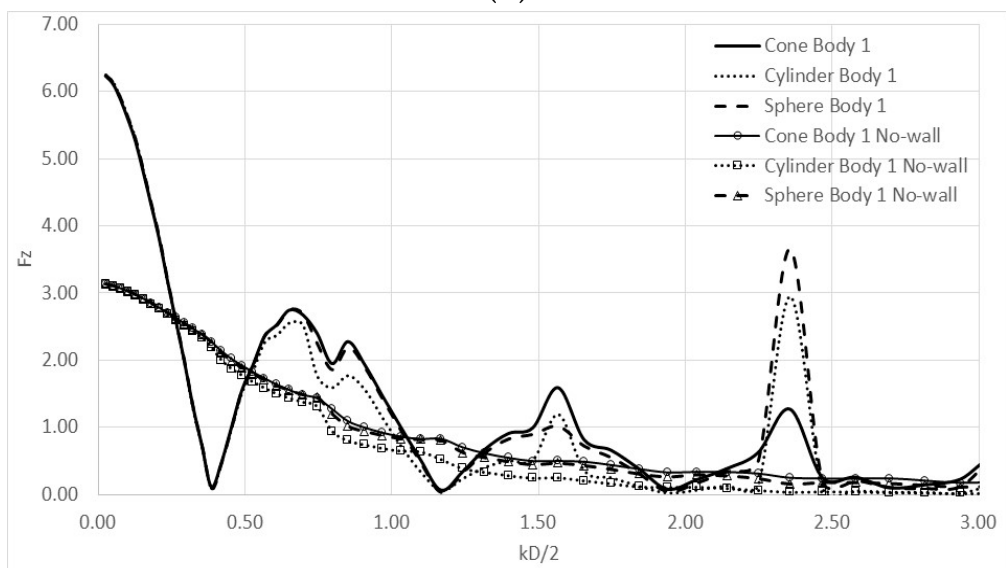

(c)

Figure 9. Dimensionless vertical exciting wave forces acting on the 1st floater of the arrays against $\mathrm{kD} / 2$, for the three different examined array configurations. Comparison with the heave exciting wave forces on the same floater of the arrays without the presence of the breakwater: (a) parallel arrangement, $\mathrm{C}_{1}$; (b) rectangular arrangement, $\mathrm{C}_{2} ;(\mathbf{c})$ perpendicular arrangement, $\mathrm{C}_{3}$.

In addition, perpendicular arrangement due to the position of the floaters with respect to the incoming wave. In addition, it can be obtained from Figure $8 \mathrm{~b}, \mathrm{c}$ that the minimization of the horizontal exciting forces does not occur at the same values of $\mathrm{kD} / 2$ (i.e., wave frequencies) as in the case of the 1st floater of the corresponding arrays, see Figure 7. It can be derived that at $\mathrm{kD} / 2=0.38 ; 0.8$; $1.17 ; \ldots$ etc. for the rectangular array and at $\mathrm{kD} / 2=0.15 ; 0.32 ; 0.45 ; \ldots$ etc. for the perpendicular 
array, the wavelength equals to a multiple value of the distance between the initial 3rd floater and its image floater. Also, the sharp peaks observed in the surge exciting forces in Figure 8 can be attributed explicitly to the waves reflected by the wall since they disappear when the wall is removed.

In Figure 9, the heave exciting wave forces on the 1st floater are plotted for every examined array configuration. It is notable that for $\mathrm{kD} / 2$ tending to zero, the heave exciting forces on the floater are almost two times larger than the forces on the same floater of the array without the presence of the breakwater. Furthermore, it can be seen that the heave forces minimize at several wave frequencies (i.e., $\mathrm{kD} / 2=0.38 ; 1.17 ; \ldots$ etc.). This behavior does not appear in the cases of the arrays without the existence of the vertical wall. The zeroing of the heave exciting force is due to the interaction phenomena between the floaters and the breakwater and in particular it appears when the distance between the initial 1st floater and its image device equals to a multiple value of the half wave length [47]. The same conclusions can be drawn also from the Figure 10, concerning the double values of the heave exciting forces at $\mathrm{kD} / 2$ tending to zero, as well as the minimization of the loads at wave frequencies corresponding to distances, between the initial and image floater, equal to the half of the wave length. Following the conclusions of [48] the duplication of the heave exciting forces when $\mathrm{kD} / 2$ tends to zero can be traced back to the fact that a fully wave-reflecting wall of infinite length has been considered here. However, this would not be the case if a finite length breakwater was examined. It can be also seen from the Figures 9 and 10 that, similar to the surge exciting forces, the sharp peaks observed in heave exciting forces can be attributed to the waves reflected by the wall since they disappear when the wall is removed.

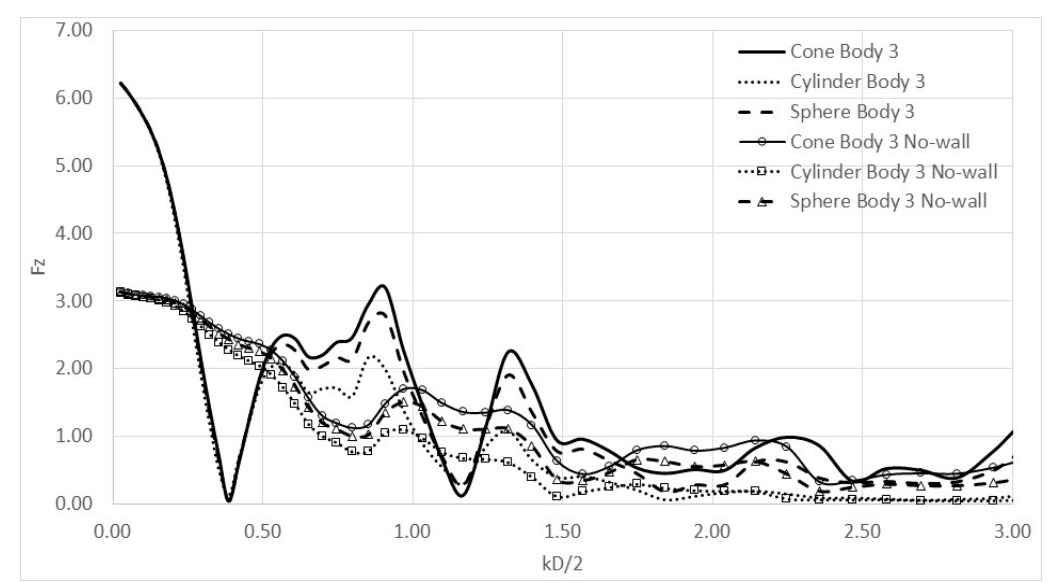

(a)

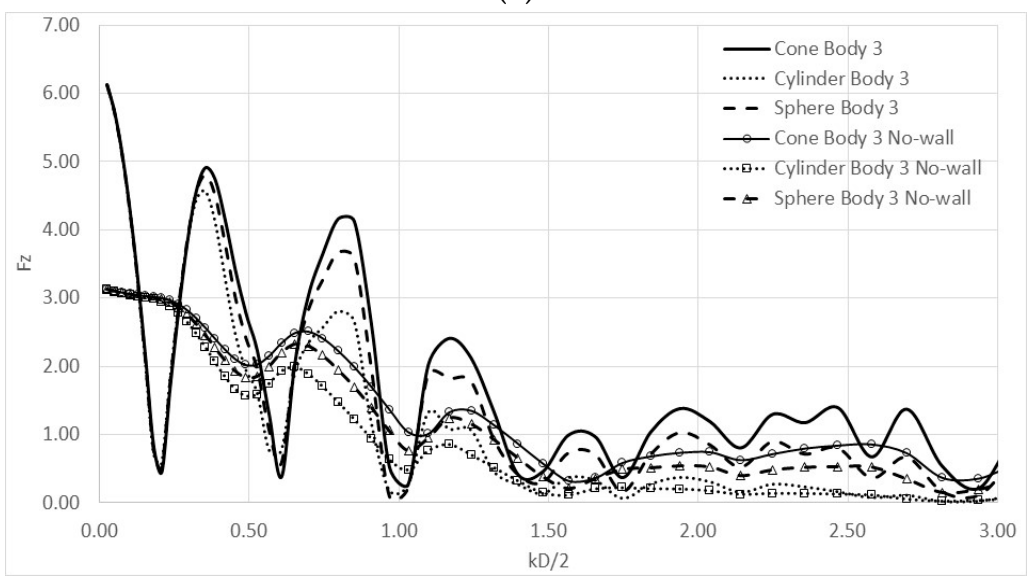

(b)

Figure 10. Cont. 


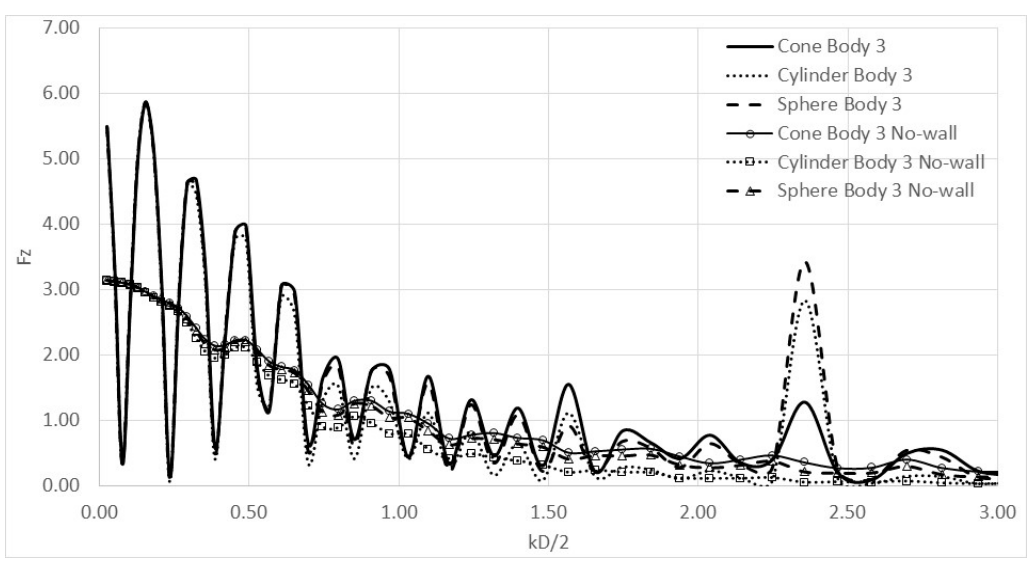

(c)

Figure 10. Dimensionless vertical exciting wave forces acting on the 3rd floater of the arrays against $\mathrm{kD} / 2$, for the three different examined array configurations. Comparison with the heave exciting wave forces on the same floater of the arrays without the presence of the breakwater: (a) parallel arrangement, $\mathrm{C}_{1} ;(\mathbf{b})$ rectangular arrangement, $\mathrm{C}_{2} ;(\mathbf{c})$ perpendicular arrangement, $\mathrm{C}_{3}$.

Next, the hydrodynamic coefficients of the floaters for the examined array configurations (see Figure 6) are presented against kD/2. More specifically, the hydrodynamic added mass and the damping coefficients as they have been derived by Equation (19) are plotted in a dimensionless form i.e., $A_{i j q p}=\frac{a_{i, j}^{q, p}}{\frac{\rho \mathrm{D}^{3}}{8}} ; B_{i j q p}=\frac{\beta_{i, j}^{q, p}}{\frac{\omega \rho \mathrm{D}^{3}}{8}} ;$ for $i, j \leq 3 ; A_{i j q p}=\frac{a_{i, j}^{q, p}}{\frac{\rho \mathrm{D}^{4}}{16}} ; B_{i j q p}=\frac{\beta_{i, j}^{q, p}}{\frac{\omega \rho \mathrm{D}^{4}}{16}} ;$ for $i \leq 3$ and $j>3$ or $i>3$ and $j \leq 3$; and $A_{i j q p}=\frac{a_{i, j}^{q, p}}{\frac{\rho \mathrm{D}^{5}}{32}} ; B_{i j q p}=\frac{\beta_{i, j}^{q, p}}{\frac{\omega \rho \mathrm{D}^{5}}{32}} ;$ for $i, j>3$. The results are also compared with the corresponding hydrodynamic characteristics of the same floater of the array, without the presence of the breakwater (i.e., no-wall cases in the presented figures).

In Figure 11, the surge hydrodynamic added mass of the 1st floater due to its forced oscillation in the surge direction is presented for the three array configurations (i.e., parallel; rectangular and perpendicular) and compared with the corresponding values of the added masses of the same floater of the array without the presence of the breakwater. It can be seen that the values of the added mass for the three examined floaters oscillate around the corresponding values referred to the same arrays but without the breakwater. Moreover, it can be observed that for the selected position of the floater in the array (i.e., examined array configuration), a small effect on the added mass coefficient can be reported since the values of the added mass of each floater at each examined array configuration are, in general, quite similar. On the other hand, the type of the floater appears to have a major impact on its added mass. It can be seen that the surge added mass of the cylindrical floater has the larger values compared with the ones of the conical and the semi-spherical floater, due to the cylinder's larger volume. Moreover, it should be also noted that as far as the vertical arrangement is concerned, the interaction phenomena between the cylindrical floaters, oscillating in the surge direction, and the breakwater create a negative added mass at $\mathrm{kD} / 2=1.17$. However, this is not the case for the conical and the semi-spherical floater.

In Figure 12, the damping coefficient of the 1st floater due to its forced oscillation in the surge direction is presented for the three aforementioned array configurations and floater types (see Figures 1 and 6). The results are also compared with the corresponding damping coefficients referred to the same floater of the arrays, without the presence of the breakwater. It can be seen from the Figure 12, that the breakwater affects also the damping coefficients. The values of the damping coefficients, when the floater is placed in front of the vertical wall, oscillate around those of the same floater without the presence of the wall. Moreover, it can be observed that the damping coefficient maximizes at the same wave frequency (i.e., $\mathrm{kD} / 2=1.17$ ) where the surge exciting forces are also maximizing, regardless the 
examined array configuration. As far as the comparison of the values of the damping coefficient of the examined types of floaters is concerned, it can be seen that due to its larger volume the cylindrical floater is characterized by higher damping coefficients compared with the ones from the conical and the semi-spherical floater.

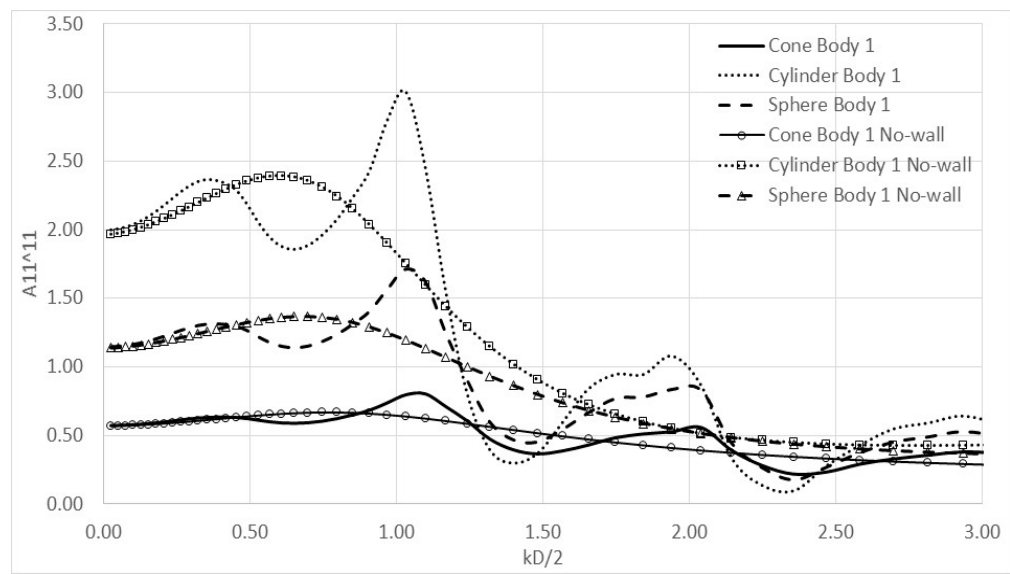

(a)

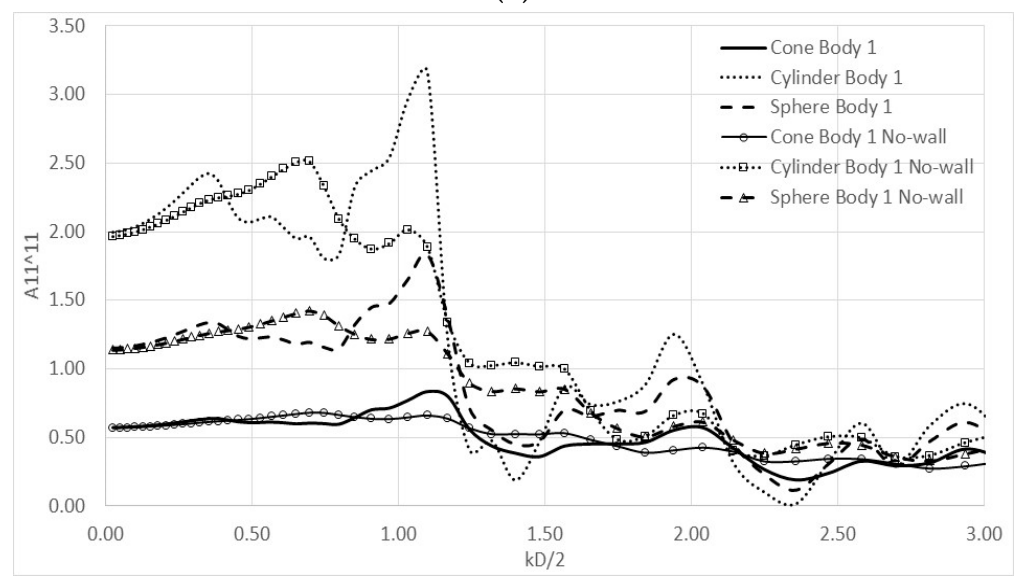

(b)

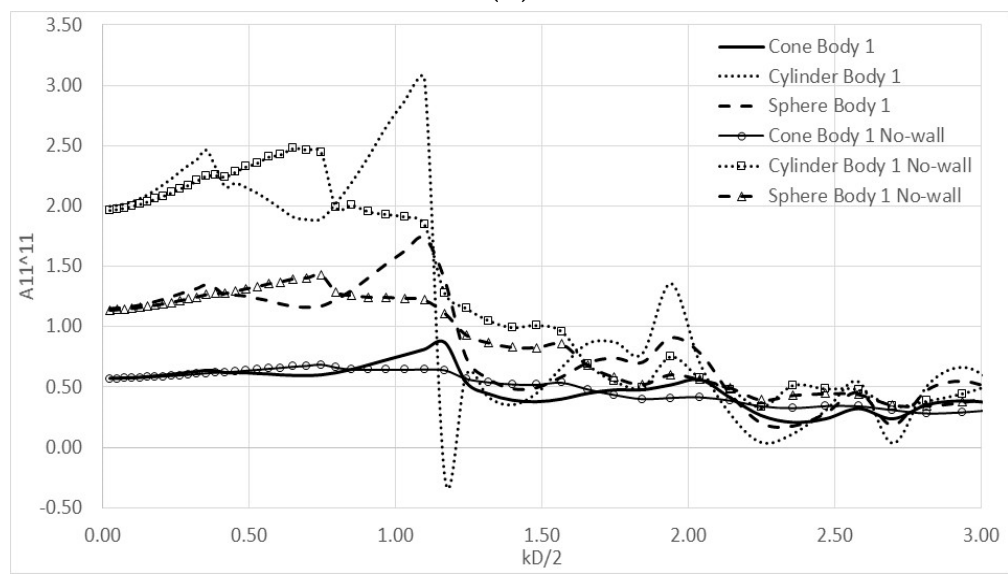

(c)

Figure 11. Dimensionless added mass of the 1st floater at the surge direction due to its motion at surge against kD/2, for the three different examined array configurations. Comparison with the corresponding added mass on the same floater of the arrays without the presence of the breakwater: (a) parallel arrangement, $\mathrm{C}_{1} ;(\mathbf{b})$ rectangular arrangement, $\mathrm{C}_{2} ;(\mathbf{c})$ perpendicular arrangement, $\mathrm{C}_{3}$. 


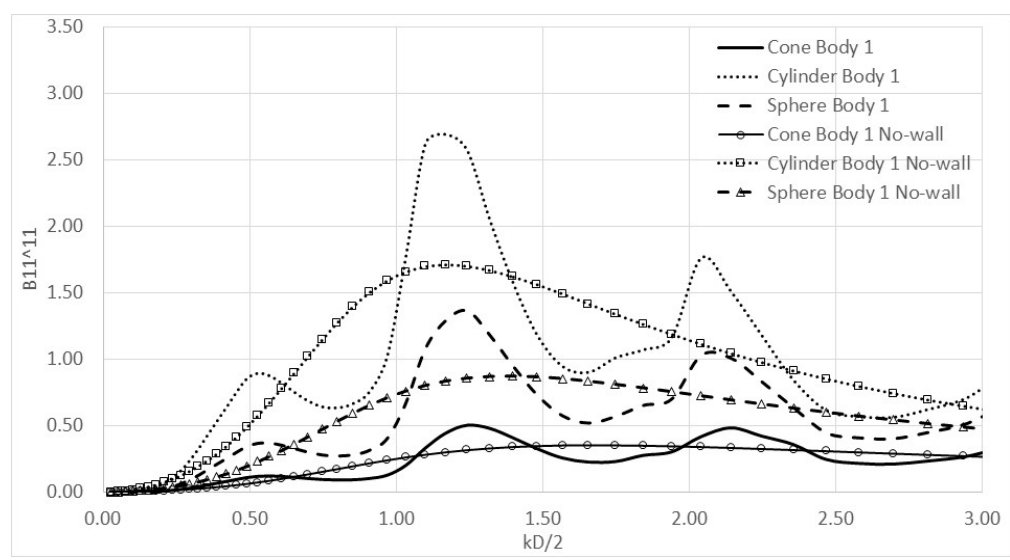

(a)

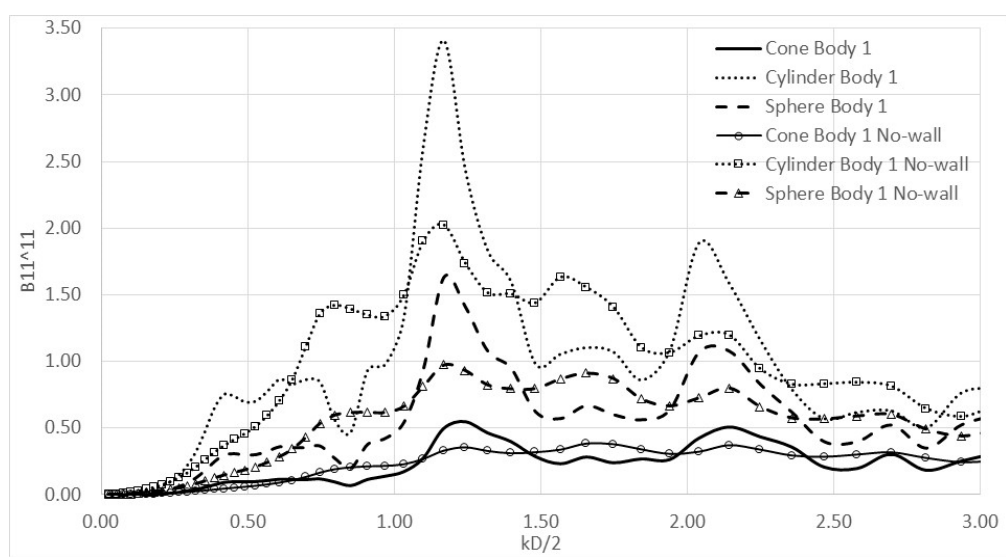

(b)

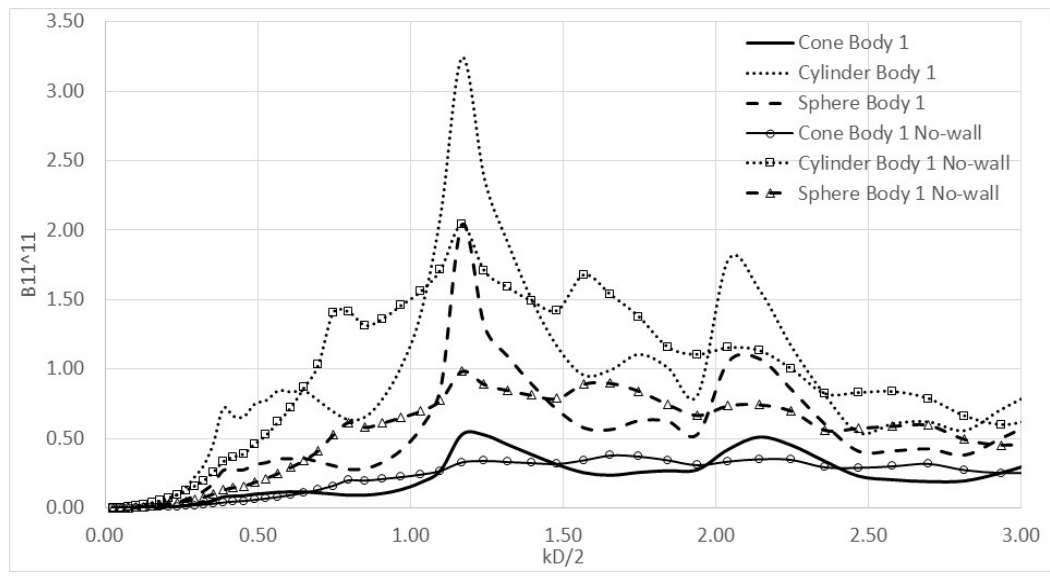

(c)

Figure 12. Dimensionless damping coefficient of the 1st floater at the surge direction due to its motion at surge against $\mathrm{kD} / 2$, for the three different examined array configurations. Comparison with the corresponding damping coefficient of the same floater of the arrays without the presence of the breakwater: (a) parallel arrangement, $\mathrm{C}_{1}$; (b) rectangular arrangement, $\mathrm{C}_{2} ;$ (c) perpendicular arrangement, $\mathrm{C}_{3}$.

In Figures 13 and 14, the corresponding surge added mass and damping coefficient of the 3rd floater of the examined array configurations (see Figure 6), due to its surge forced oscillations are presented. Herein, the results are compared also with the corresponding coefficients of the same floater of the array but with absence of the breakwater. 


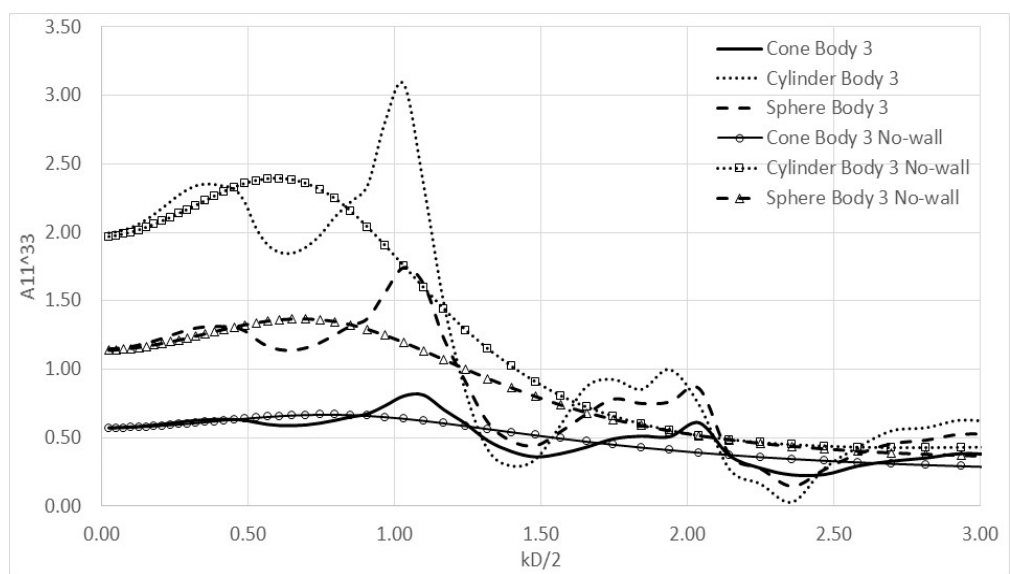

(a)

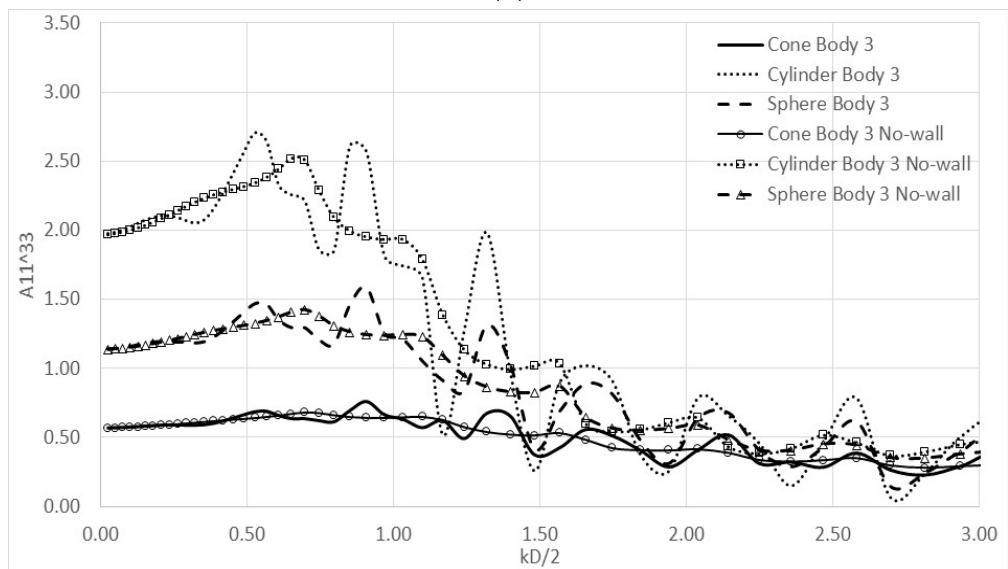

(b)

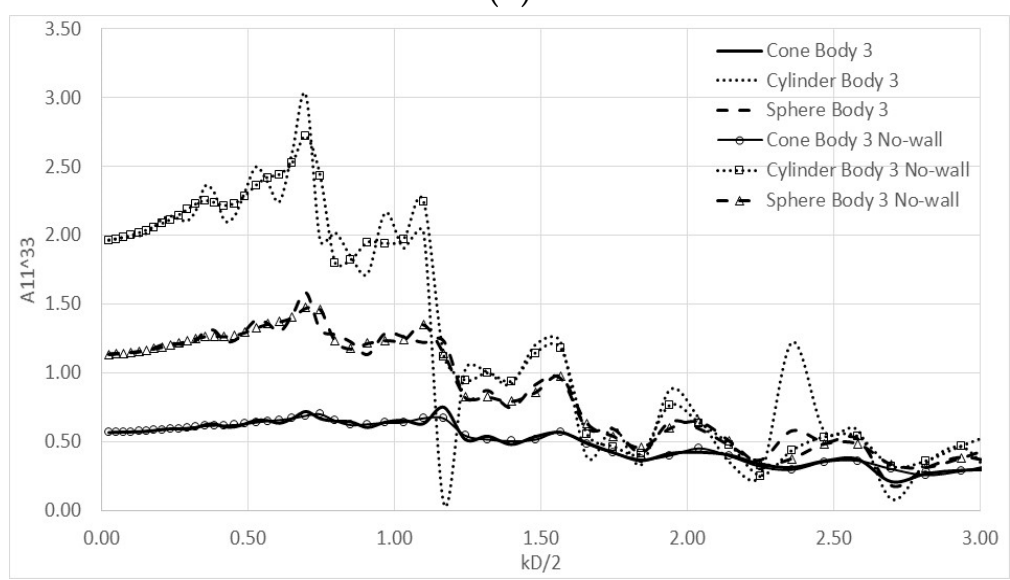

(c)

Figure 13. Dimensionless added mass of the 3rd floater at the surge direction due to its motion at surge against kD/2, for the three different examined array configurations. Comparison with the corresponding added mass on the same floater of the arrays without the presence of the breakwater: (a) parallel arrangement, $\mathrm{C}_{1} ;(\mathbf{b})$ rectangular arrangement, $\mathrm{C}_{2} ;(\mathbf{c})$ perpendicular arrangement, $\mathrm{C}_{3}$. 


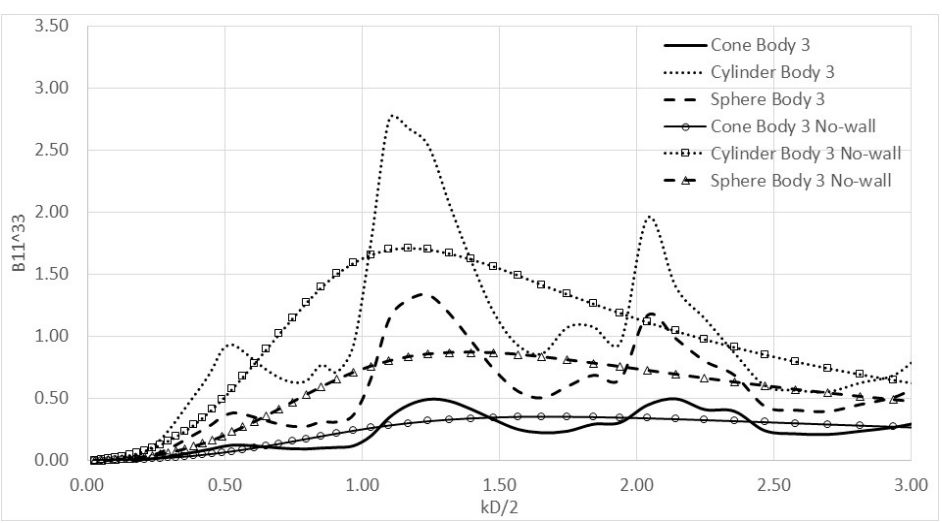

(a)

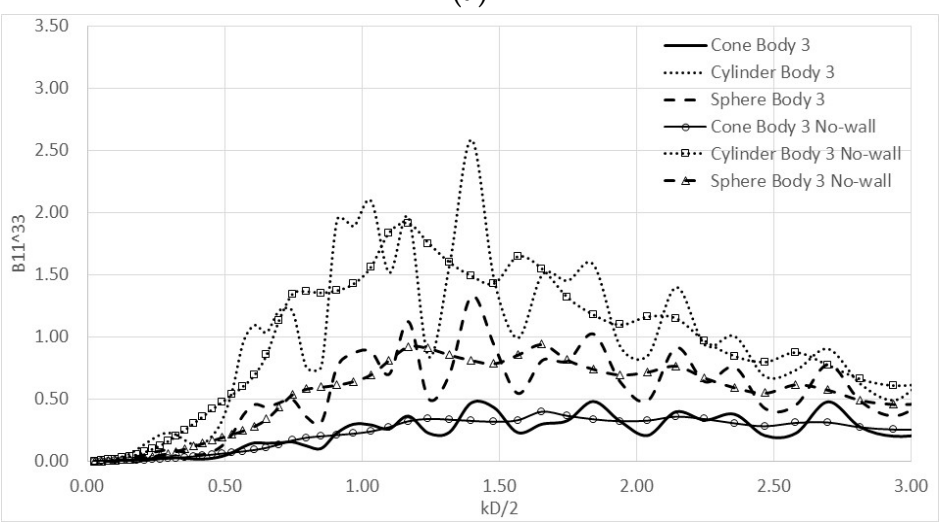

(b)

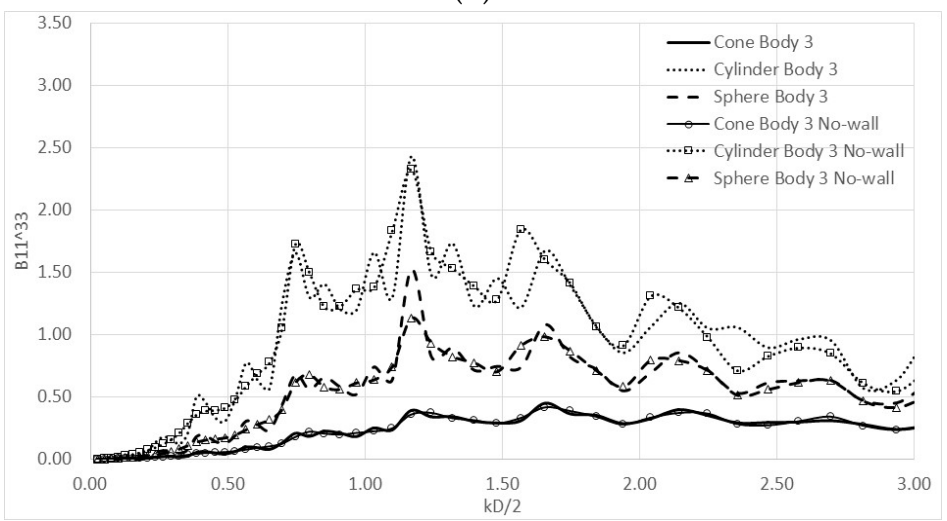

(c)

Figure 14. Dimensionless damping coefficient of the 3rd floater at the surge direction due to its motion at surge against $\mathrm{kD} / 2$, for the three different examined array configurations. Comparison with the corresponding damping coefficient of the same floater of the arrays without the presence of the breakwater: (a) parallel arrangement, $\mathrm{C}_{1} ;(\mathbf{b})$ rectangular arrangement, $\mathrm{C}_{2} ;$ (c) perpendicular arrangement, $\mathrm{C}_{3}$.

Comparing the Figures 11a and 13a it can be seen that the position of each floater in the parallel array configuration does not affect the added mass in surge direction, since both figures (i.e., added mass of the 1st and the 3rd floater) depict very similar results. Furthermore, in these figures the effect of the breakwater is also notable since the values of the added mass of the floater placed in front of a breakwater oscillate around those without the presence of the vertical wall. However, this is not occurring in the cases of the perpendicular and the rectangular arrangements (see Figure $11 \mathrm{~b}, \mathrm{c}$ and Figure 13b,c). The effect of the breakwater on the added mass of the 3rd floater is decreasing for these two arrangements, since the oscillations of the values of the floater's added mass placed in front of the 
wall, around those of the same floater without the presence of the wall, decrease. This is happening because the 3rd floater is closer to the wall at the parallel array, whereas this distance increases for the rectangular and vertical arrangement. The same conclusion can be drawn for the damping coefficients of the 3rd floater presented in Figure 14. Following the remarks by [49], the arrays of WECs can be divided into broad categories, based on the converters spacing relative to the wavelength. When the ratio of the spacing between the converters of the array and the radius of the WEC has a value larger than the wavelength the interaction phenomena between the converters decrease significantly and each device tends to behave as a single converter. From the image theory presented in this work, the system of the $N$ converters and the breakwater has been simulated as an array of $2 N$ converters consisting of the initial and their image virtual devices with respect to the breakwater that are exposed to the action of surface waves (diffraction problem) or forced to move in otherwise calm water (radiation problems) without, however, the presence of the wall. At the examined perpendicular arrangement this ratio is larger than the wavelength for $\mathrm{kD} / 2>0.7$. However, for lower values of $\mathrm{kD} / 2$, where the ratio is tending to the wave length, the interaction phenomena on the initial device due to the motion of its image device are also small. Thus, the breakwater seems to have minor effect on the added mass and on the damping coefficient of the 3rd floater of the array.

Concluding, as far as the surge added mass and damping coefficients are concerned, their values seem to be mainly dictated by the type of the floater (i.e., larger coefficients appear for the cylindrical floater and follow the semi-spherical and the conical floater) and not to a large extend by the floater's array configuration.

In the sequel, the heave hydrodynamic added masses and the heave damping coefficients of the same floaters (i.e., 1st and 3rd, see Figure 6) of the aforementioned array configurations (i.e., parallel, rectangular and vertical arrangement) are presented in Figures 15-18. The Figures 15 and 16 depict the added mass and damping coefficient, respectively, of the 1st floater of the examined array configurations, in heave direction due to its forced oscillation also in heave. Herein, the impact of the floater's type on its hydrodynamic coefficients (i.e., added mass and damping) is notable. The cylindrical floater is characterized by lower values of damping coefficients, compared to the conical and semi-spherical floater, at every examined arrangement. This was not the case for the surge damping coefficient, as being depicted in Figures 12 and 14. Furthermore, it can be observed from the Figures 15 and 16 that the effect of the breakwater is higher for small values of $\mathrm{kD} / 2$ (i.e., $\mathrm{kD} / 2<1.17$ ). For values of $\mathrm{kD} / 2$ larger than 1.17 the values of the added mass and damping coefficients of the floater in front of the breakwater tend to those of the same floater without the presence of the wall. In Figure 16 it can be also seen the doubling of the damping coefficient at values of $\mathrm{kD} / 2$ tending to zero. This has been also observed at the heave exciting forces on the floaters in front of the vertical wall.

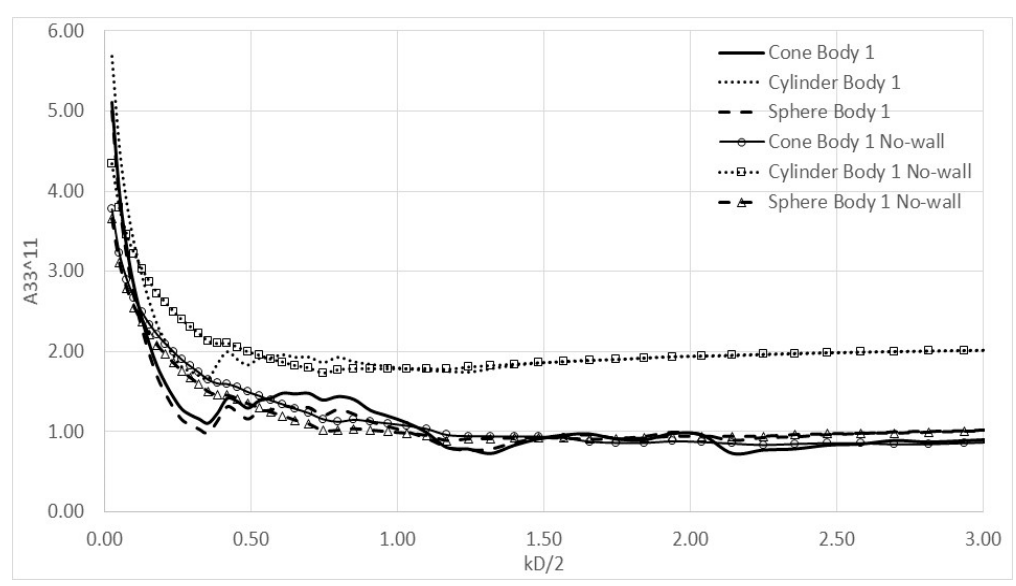

(a)

Figure 15. Cont. 


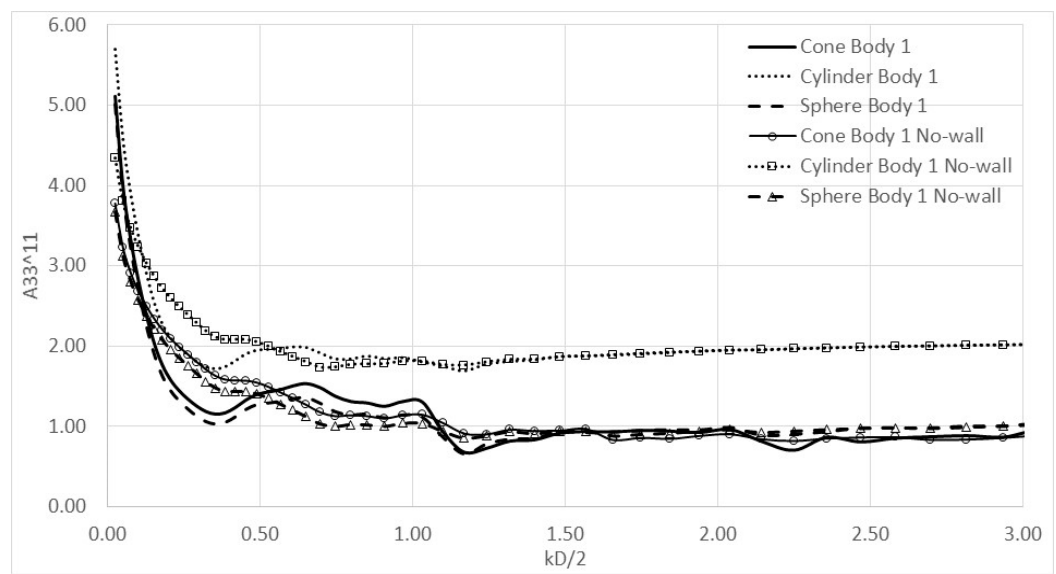

(b)

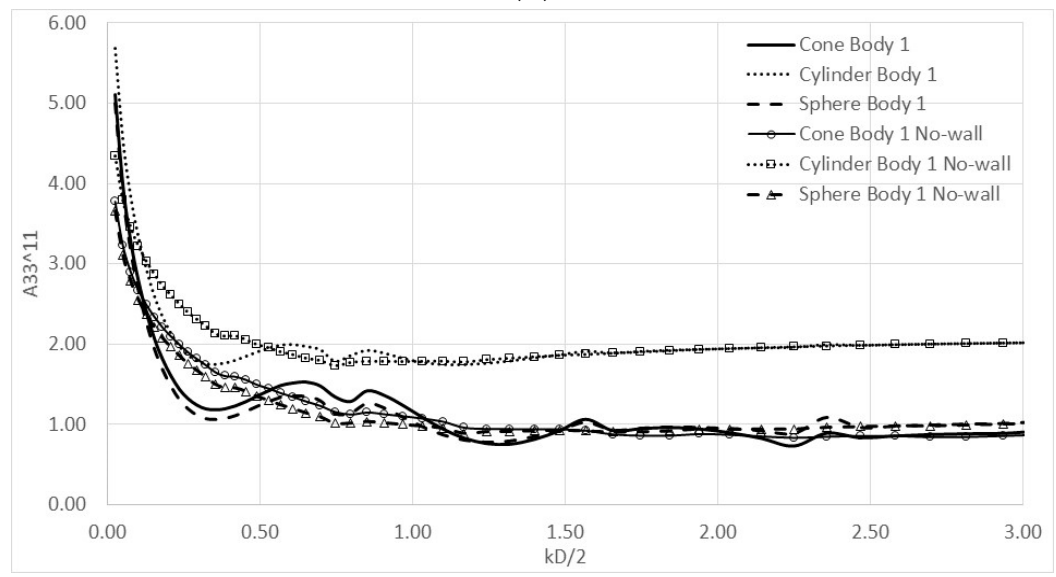

(c)

Figure 15. Dimensionless added mass of the 1st floater in the heave direction due to its motion in heave against $\mathrm{kD} / 2$, for the three different examined array configurations. Comparison with the corresponding added mass on the same floater of the arrays without the presence of the breakwater: (a) parallel arrangement, $\mathrm{C}_{1} ;(\mathbf{b})$ rectangular arrangement, $\mathrm{C}_{2} ;(\mathbf{c})$ perpendicular arrangement, $\mathrm{C}_{3}$.

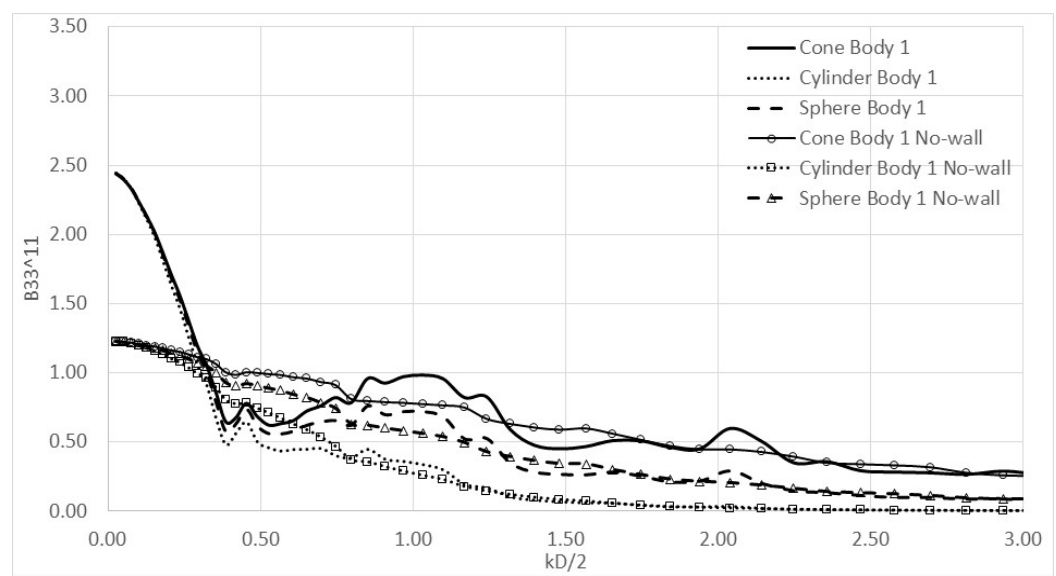

(a)

Figure 16. Cont. 


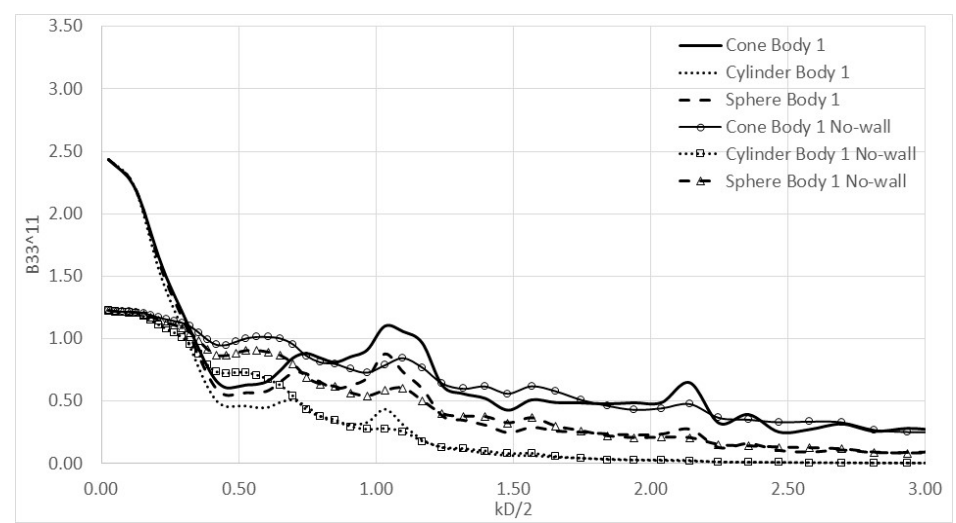

(b)

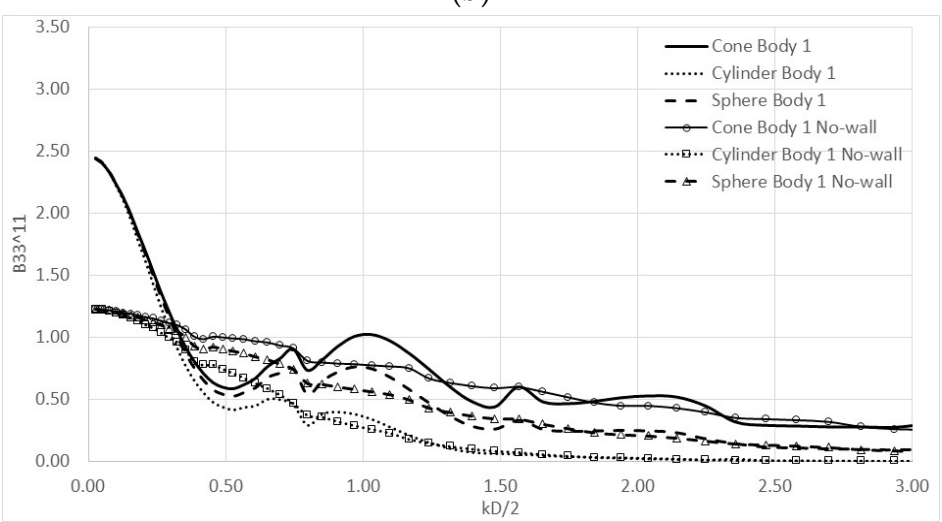

(c)

Figure 16. Dimensionless damping coefficient of the 1st floater at the heave direction due to its motion at heave against $\mathrm{kD} / 2$, for the three different examined array configurations. Comparison with the corresponding damping coefficient of the same floater of the arrays without the presence of the breakwater: (a) parallel arrangement, $\mathrm{C}_{1}$; (b) rectangular arrangement, $\mathrm{C}_{2}$; (c) perpendicular arrangement, $\mathrm{C}_{3}$.

In Figures 17 and 18, the hydrodynamic coefficients of the 3rd floater in heave (i.e., added mass and damping values) are presented for the three examined array configurations in front of the vertical wall and compared with the corresponding values of the same floater of the arrays, without the presence of the vertical wall. Comparing the Figures 15 and 16 with the Figures 17 and 18 it can be seen that the added mass and the damping coefficients of the 3rd floater in the parallel arrangement tend to those of the 1st floater, for the same array configuration.

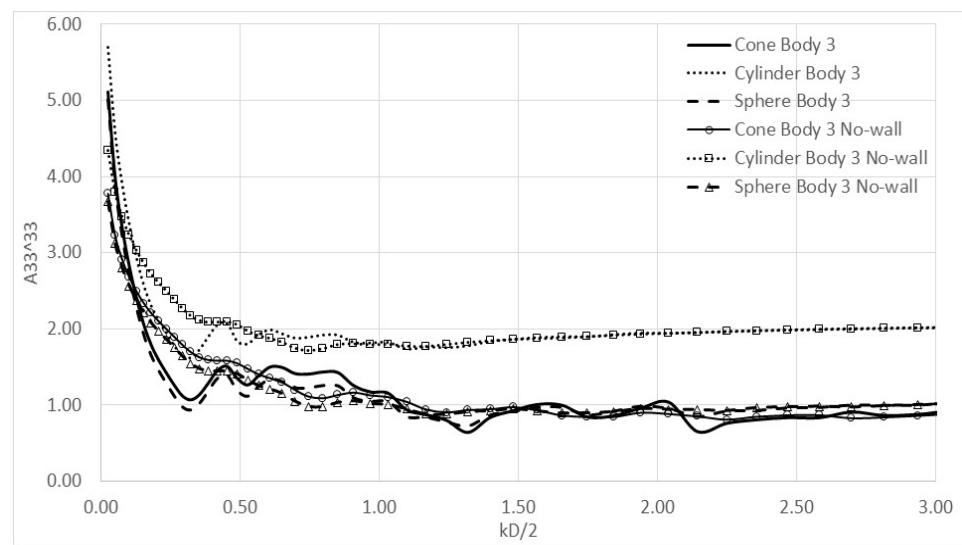

(a)

Figure 17. Cont. 


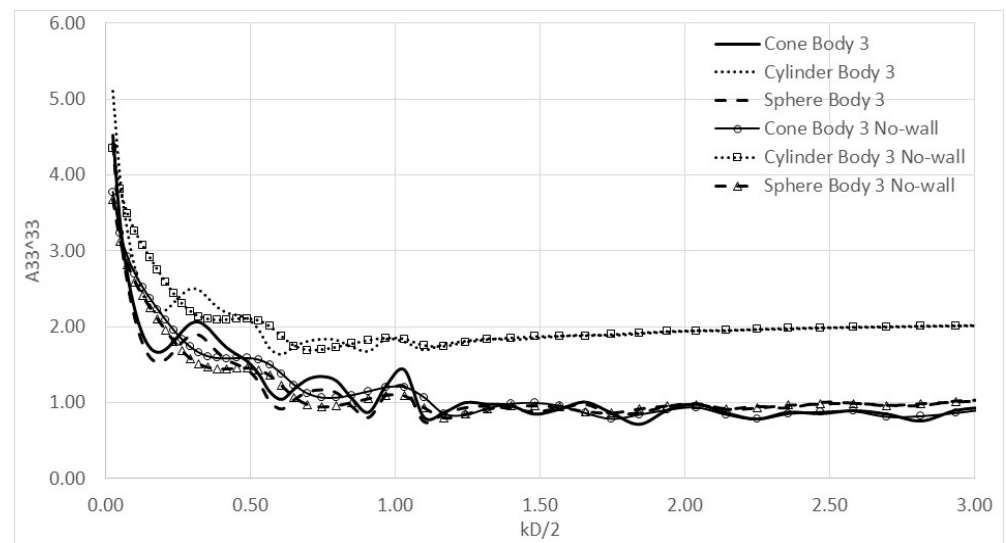

(b)

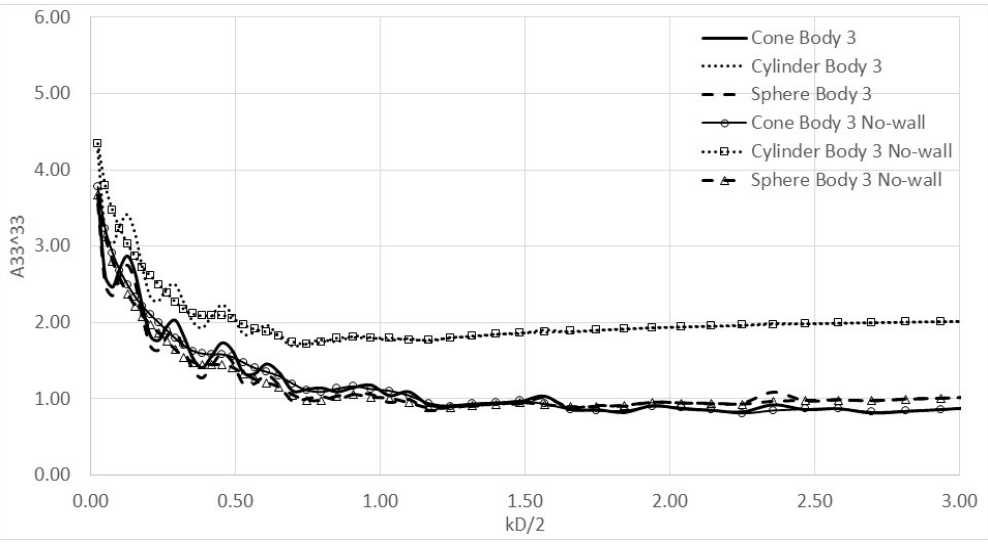

(c)

Figure 17. Dimensionless added mass of the 3rd floater at the heave direction due to its motion at heave against $\mathrm{kD} / 2$, for the three different examined array configurations. Comparison with the corresponding added mass on the same floater of the arrays without the presence of the breakwater: (a) parallel arrangement, $\mathrm{C}_{1} ;(\mathbf{b})$ rectangular arrangement, $\mathrm{C}_{2} ;(\mathbf{c})$ perpendicular arrangement, $\mathrm{C}_{3}$.

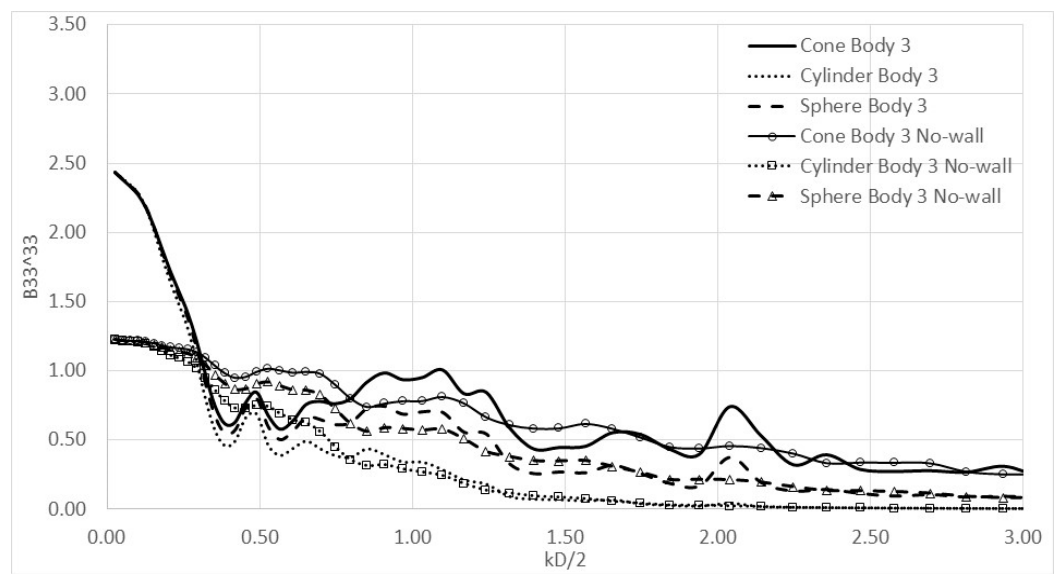

(a)

Figure 18. Cont. 


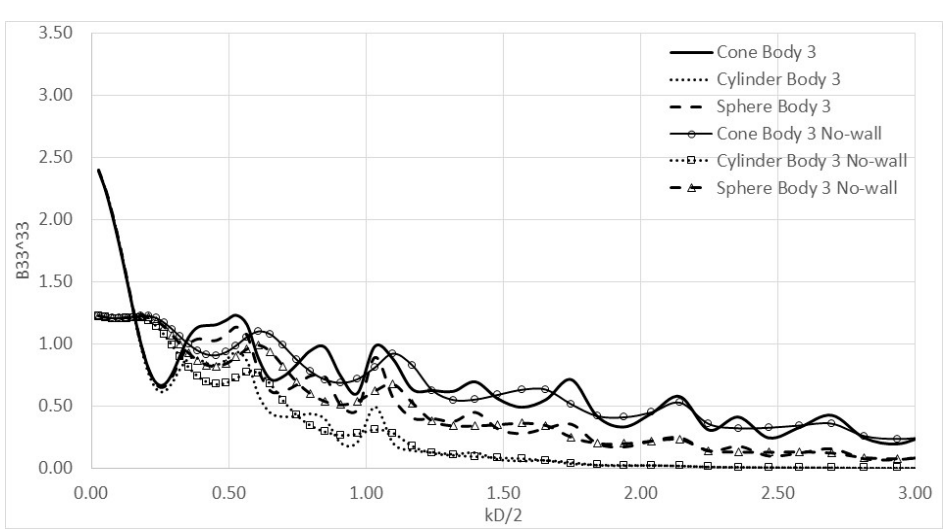

(b)

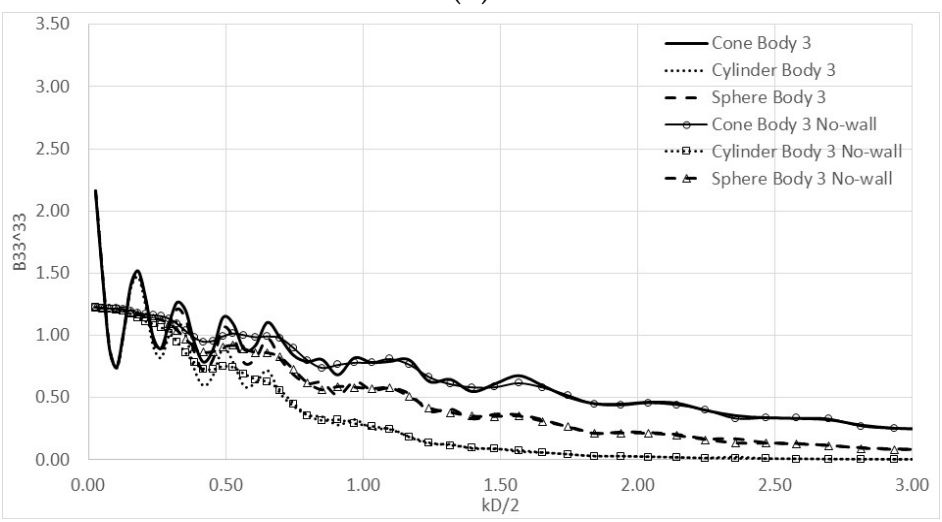

(c)

Figure 18. Dimensionless damping coefficient of the 3rd floater at the heave direction due to its motion at heave against $\mathrm{kD} / 2$, for the three different examined array configurations. Comparison with the corresponding damping coefficient of the same floater of the arrays without the presence of the breakwater: (a) parallel arrangement, $\mathrm{C}_{1} ;(\mathbf{b})$ rectangular arrangement, $\mathrm{C}_{2} ;(\mathbf{c})$ perpendicular arrangement, $\mathrm{C}_{3}$.

The same conclusion has been also drawn at the discussion of the Figures 11 and 13 . However, this is not the case for the other examined array configurations. Especially, for the perpendicular arrangement, the interaction phenomena between the floaters and the breakwater imply large oscillations of the values of the hydrodynamic coefficients of the 3rd floater, around the ones of the same floater without the presence of the vertical wall. Furthermore, it is notable that the heave hydrodynamic characteristics of the floater is mainly affected by the type of the floater (i.e., cylindrical, conical or semi-spherical) and less by its position in the array.

In the Figures 19 and 20, the hydrodynamic added mass and the damping coefficients in heave are presented for the 3rd floater of the aforementioned types (i.e., cylindrical, conical and semi-spherical) due to the forced heave oscillation of the $j$-th floater $(j=1,2, \ldots, 5)$, in the perpendicular array case configuration. The latter is selected due to the high interaction phenomena between the members of the array compared with the ones that appear in the parallel and rectangular array. In the Appendix A, these values (i.e., heave hydrodynamic added mass and damping coefficients for the 3rd floater) are also presented in a tabular form for indicative values of wave frequencies to allow more accurate comparisons to be made with other numerical estimates.

Comparing the Figure 19a-c it can be seen that the type of the floater affects mainly the heave added mass due to its own forced heave oscillation. Thus, the higher added mass is applied for the cylindrical floater followed by the added mass from the semi-spherical and conical floater. On the other hand, the added mass on the floater, due to the forced heave motion of the rest bodies of the array, seems not to be affected by the type of the floater, since the values of the hydrodynamic mass 
of the floaters have similar values. This is also the case for the damping coefficient of the 3rd floater in the heave direction due to the forced oscillation at heave of the $j$-th floater, presented in Figure 20. The contribution of the floater's shape on the damping coefficients of the 3rd floater, due to the forced heave motion of the remaining floaters, seems to be minor. However, contrary to the added mass, the higher values of damping coefficients are presented for the conical and semi-spherical floater, followed by the ones of the cylindrical floater.

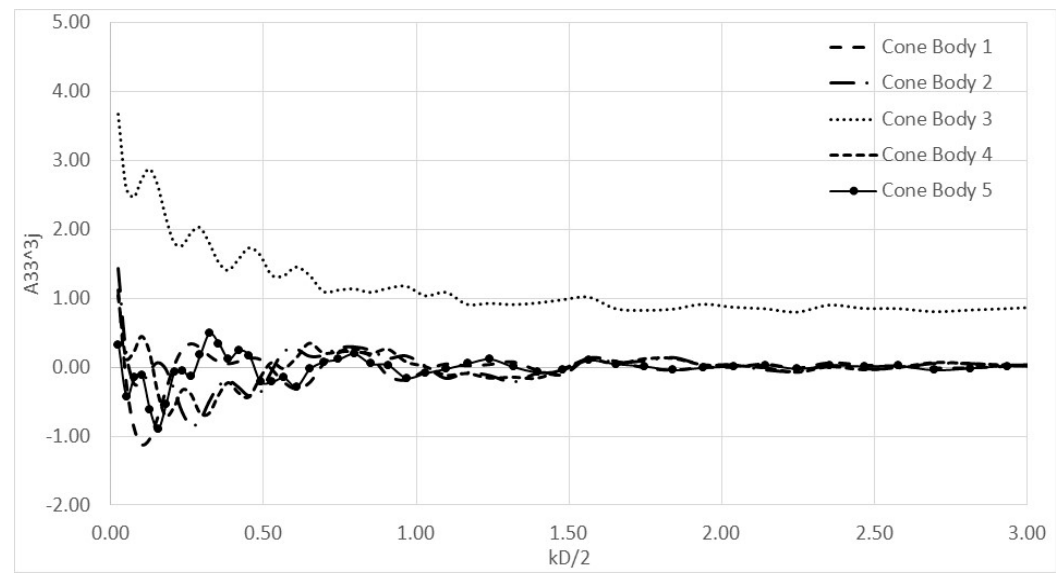

(a)

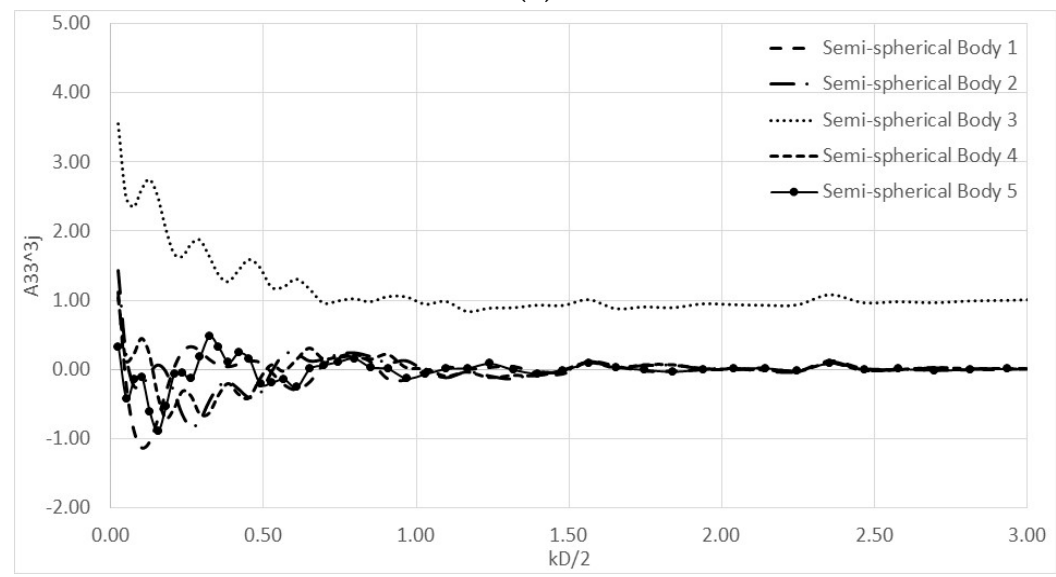

(b)

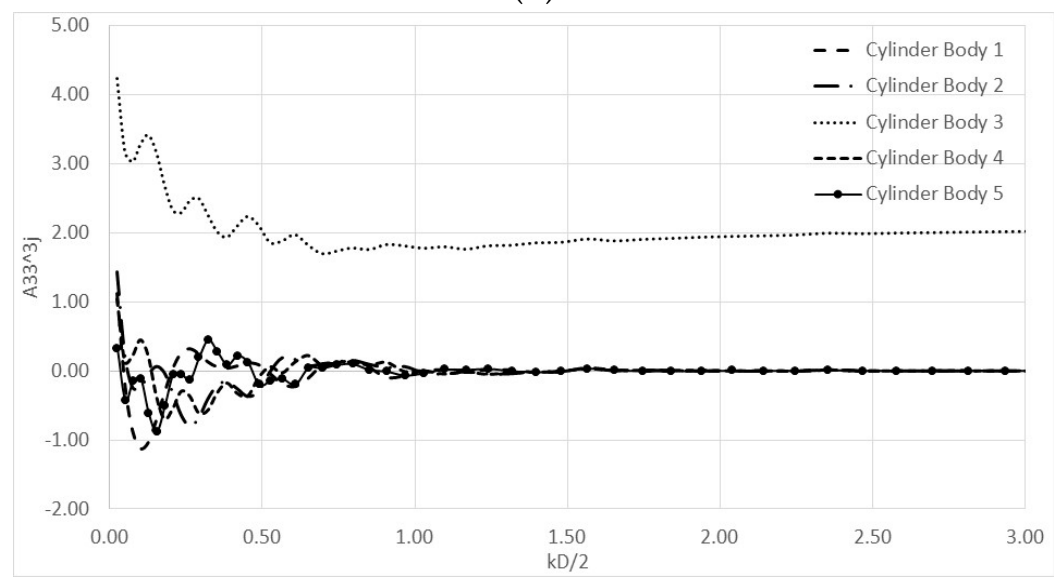

(c)

Figure 19. Dimensionless added mass of the 3rd floater in the heave direction due to the forced motion at heave of the $j$-th floater $(j=1,2, \ldots, 5)$ against $\mathrm{kD} / 2$, for the three different examined types of floaters: (a) conical floater; (b) semi-spherical floater; (c) cylindrical floater in the perpendicular arrangement, $\mathrm{C}_{3}$. 


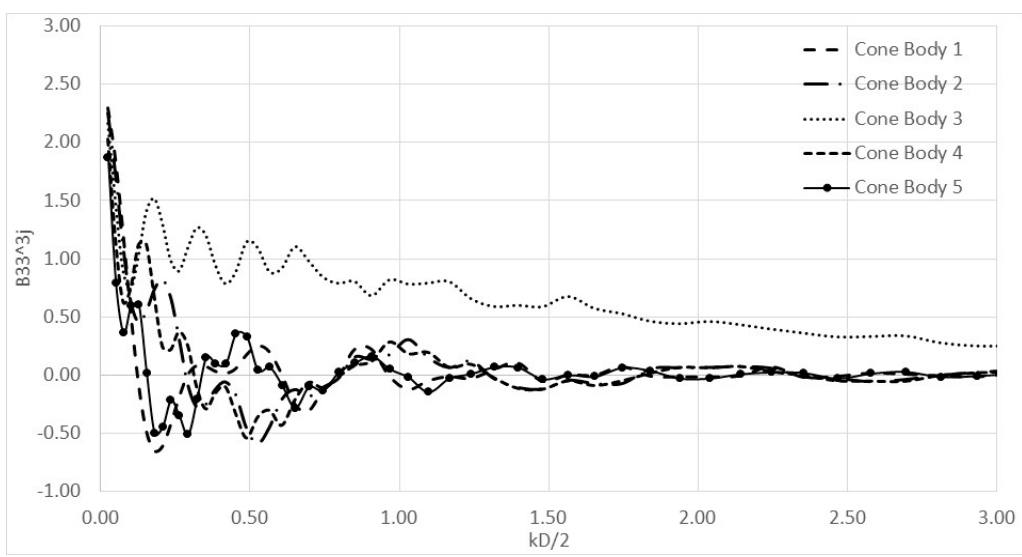

(a)

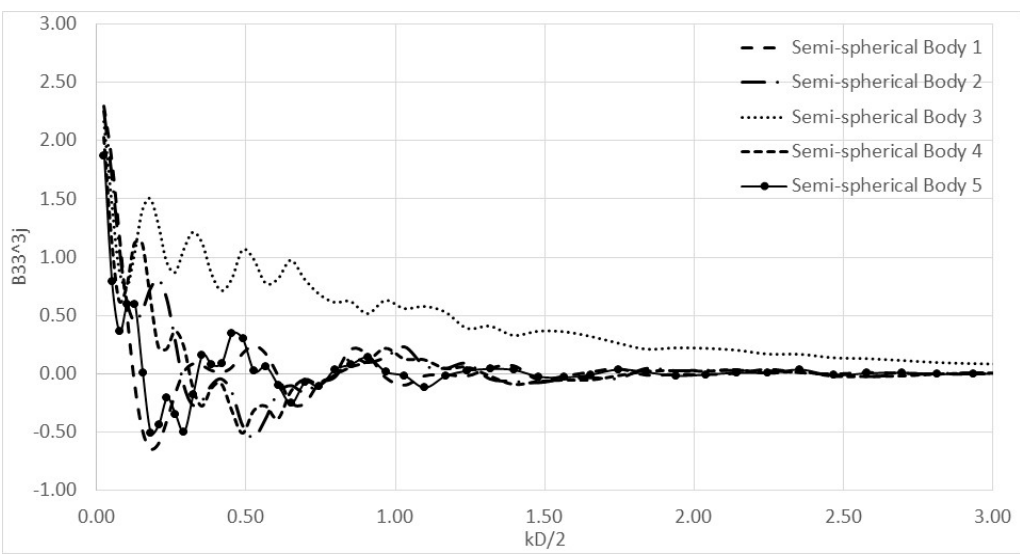

(b)

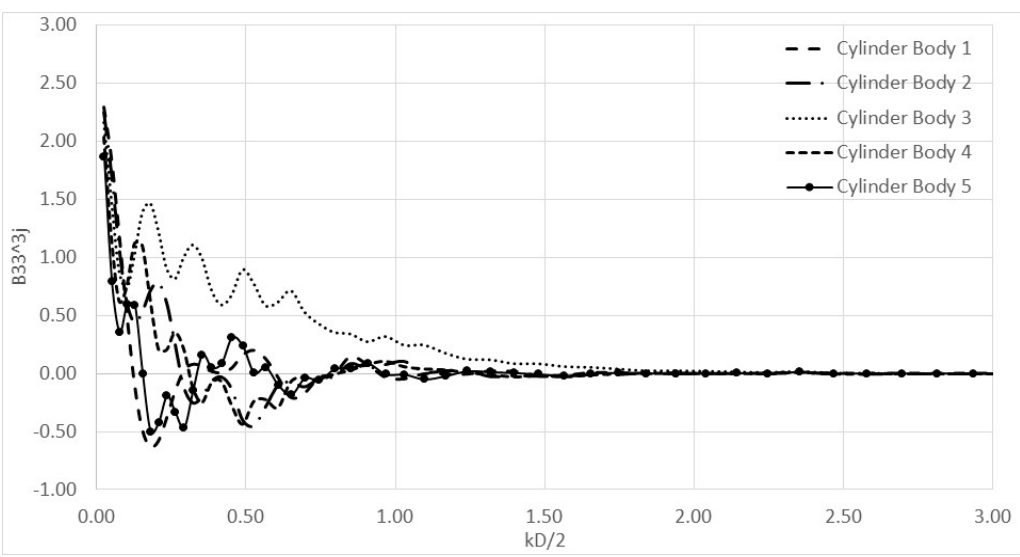

(c)

Figure 20. Dimensionless damping coefficient of the 3rd floater at the heave direction due to the forced motion at heave of the $j$-th floater $(j=1,2, \ldots, 5)$ against $\mathrm{kD} / 2$, for the three different examined types of floaters: (a) conical floater; (b) semi-spherical floater; (c) cylindrical floater in the perpendicular arrangement, $\mathrm{C}_{3}$.

In the Figures 21 and 22 the hydrodynamic added mass and the damping coefficients in sway (y-direction, see Figure 6) are presented for the 1st floater in the parallel array case configuration, $\mathrm{C}_{1}$, due to the forced sway oscillation of the $j$-th floater $(j=1,2, \ldots, 5)$ in dependence on the floater's geometry, i.e., cylindrical, conical and semi-spherical. It can be seen from the Figures 21 and 22 that the type of the floater affects its hydrodynamic characteristics not only due to its own forced sway 
oscillation but also to the forced oscillation in the sway direction of the rest of the floaters. Thus, due to its larger volume the cylindrical floater is characterized by higher hydrodynamic characteristics compared with the ones from the conical and the semi-spherical floater. Indicative theoretical results for the cylindrical floater case, i.e., Figures $21 \mathrm{c}$ and $22 \mathrm{c}$, are given in the Appendix B, (i.e., sway hydrodynamic added mass and damping coefficients for the 1st floater) in tabular form to allow more accurate comparisons to be made with other numerical estimates.

In the Figure 23 the hydrodynamic added mass and the damping coefficient in sway (y-direction, Figure 6) are presented indicatively for the 1st conical floater due to the forced surge oscillation (x-direction) of the $j$-th conical floater $(j=1,2, \ldots, 5)$, in the parallel array case configuration, $C_{1}$. The results are compared with the corresponding values of the 1st conical floater of the array without the presence of the breakwater. Due to the examined arrangement (i.e., parallel) these values (i.e., hydrodynamic added mass and damping coefficient in sway for the 1st conical floater due to the forced surge oscillation of the $j$-th conical floater) tend to zero at every wave frequency, when the vertical wall is absent. Nevertheless, this is not the case for the array placed in front of the breakwater, since it is evident from the figures that the examined hydrodynamic characteristics of the floater appear no zero values. Indicative theoretical results for the hydrodynamic interaction coefficients concerning the 1st conical floater, see Figure 23a,b, are given in the Appendix C, in tabular form to allow more accurate comparisons to be made with other numerical estimates.

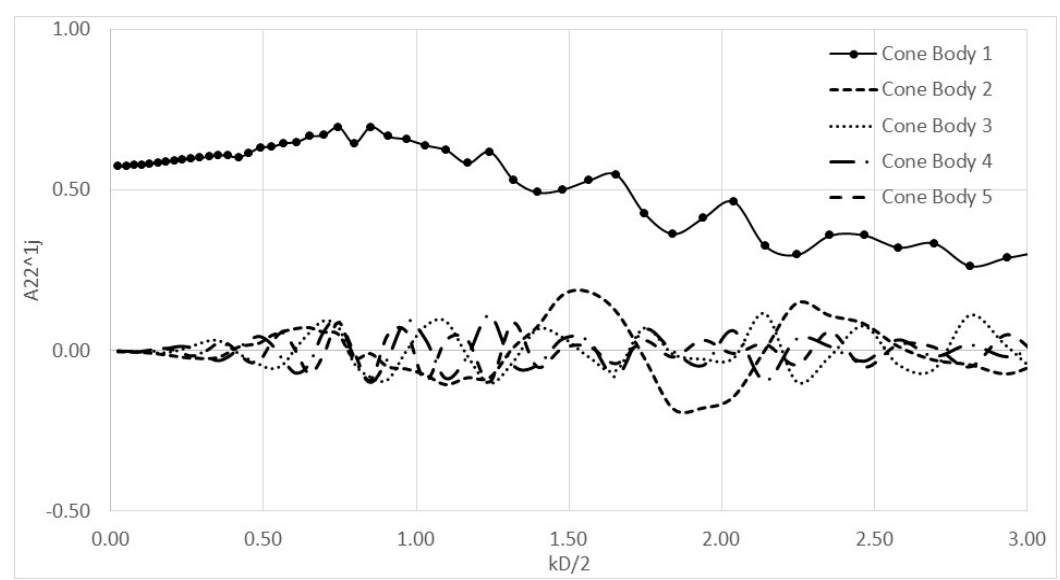

(a)

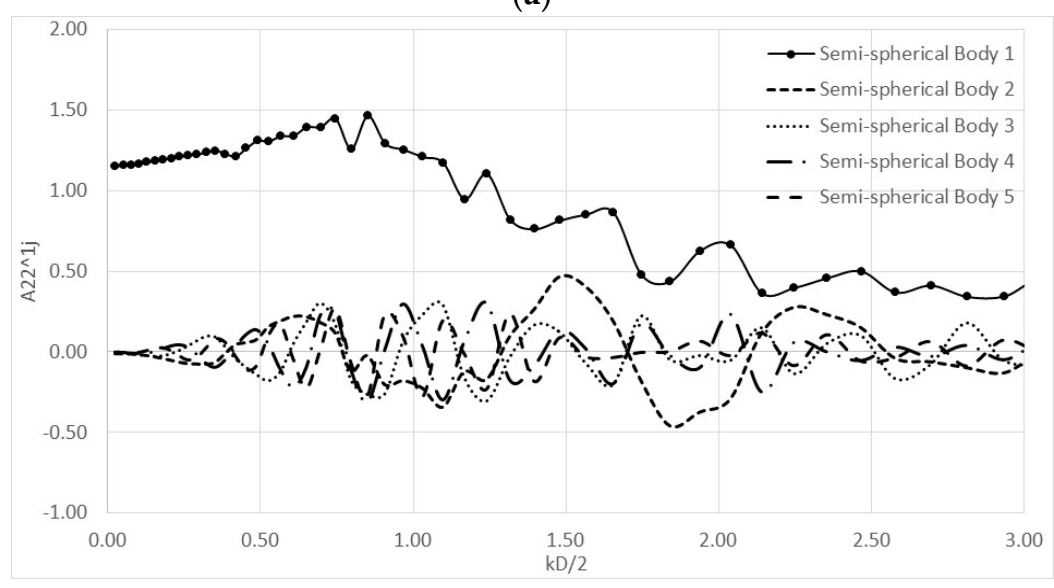

(b)

Figure 21. Cont. 


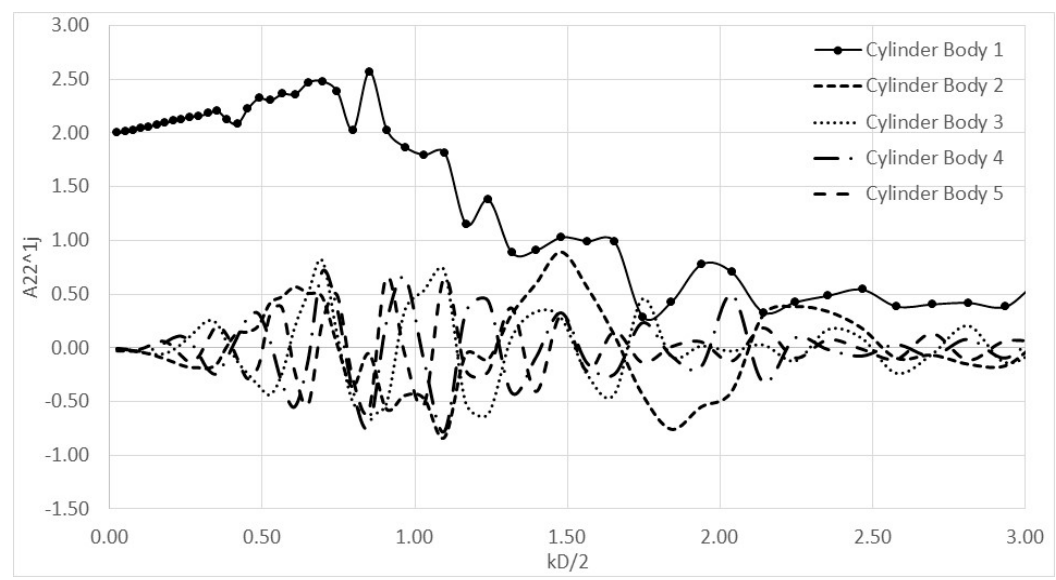

(c)

Figure 21. Dimensionless added mass of the 1st floater in the sway direction due to the forced motion in sway of the $j$-th floater $(j=1,2, \ldots, 5)$ against $\mathrm{kD} / 2$, for the three different examined types of floaters: (a) conical floater; (b) semi-spherical floater; (c) cylindrical floater in the parallel array case configuration, $\mathrm{C}_{1}$.

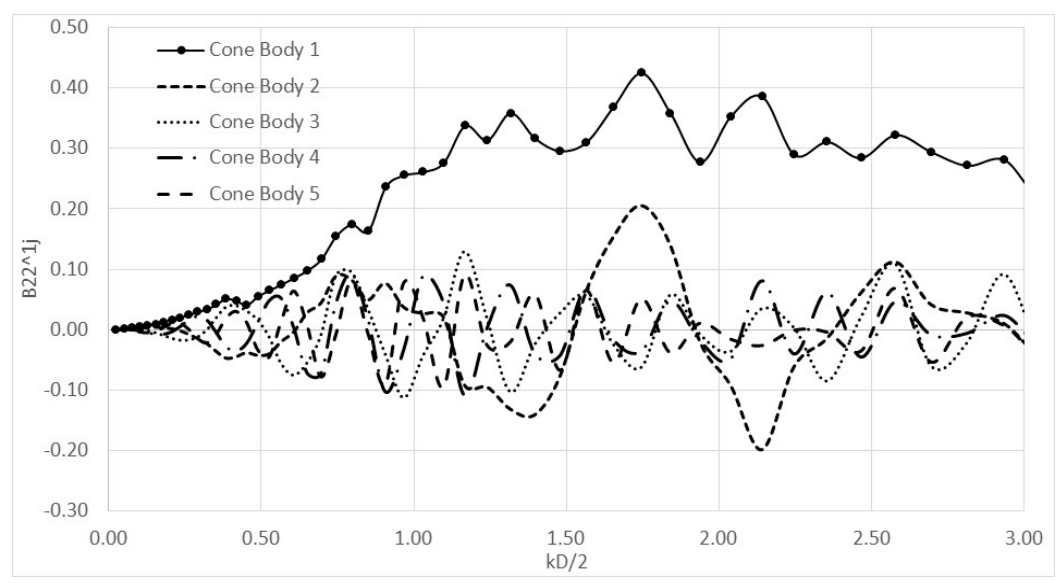

(a)

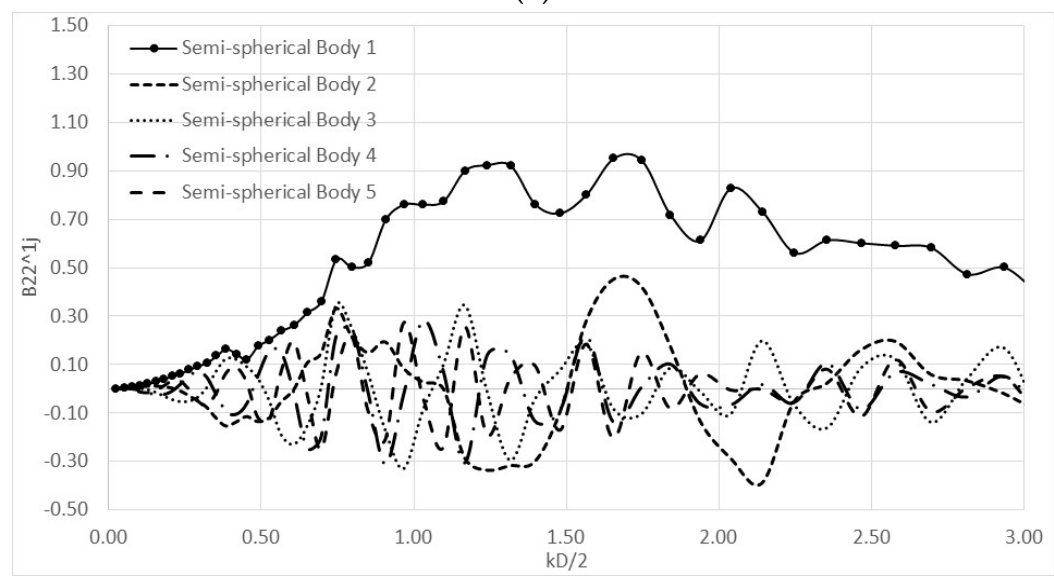

(b)

Figure 22. Cont. 


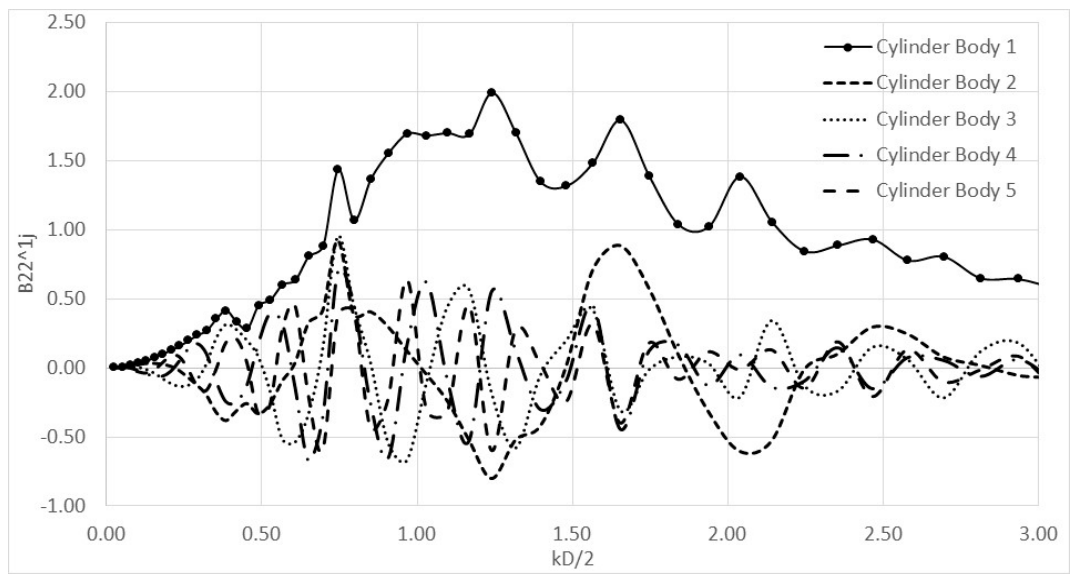

(c)

Figure 22. Dimensionless damping coefficient of the 1st floater at the sway direction due to the forced motion in sway of the $j$-th floater $(j=1,2, \ldots, 5)$ against $\mathrm{kD} / 2$, for the three different examined types of floaters: (a) conical floater; (b) semi-spherical floater; (c) cylindrical floater in the parallel array case configuration, $\mathrm{C}_{1}$.

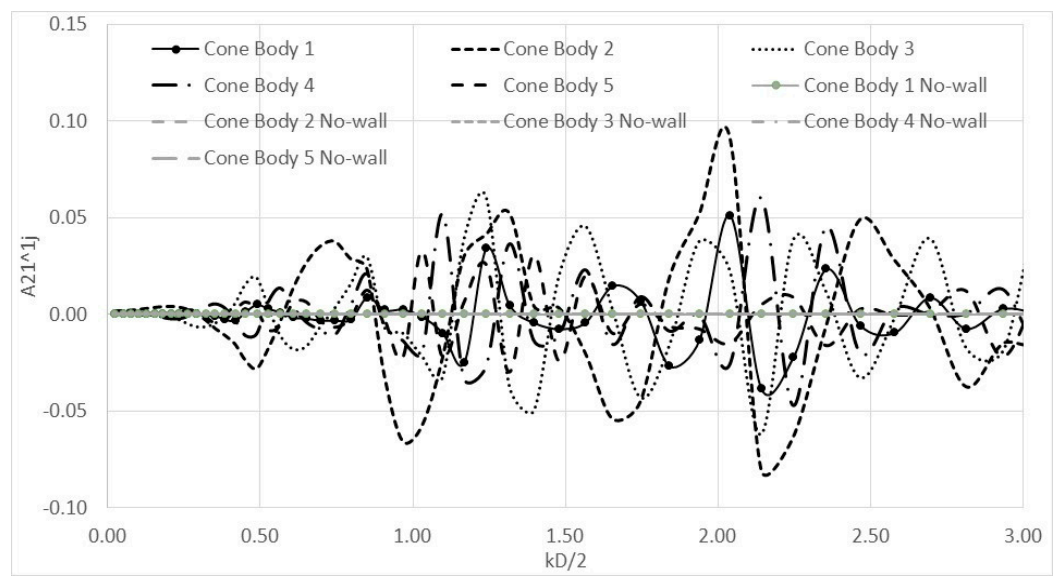

(a)

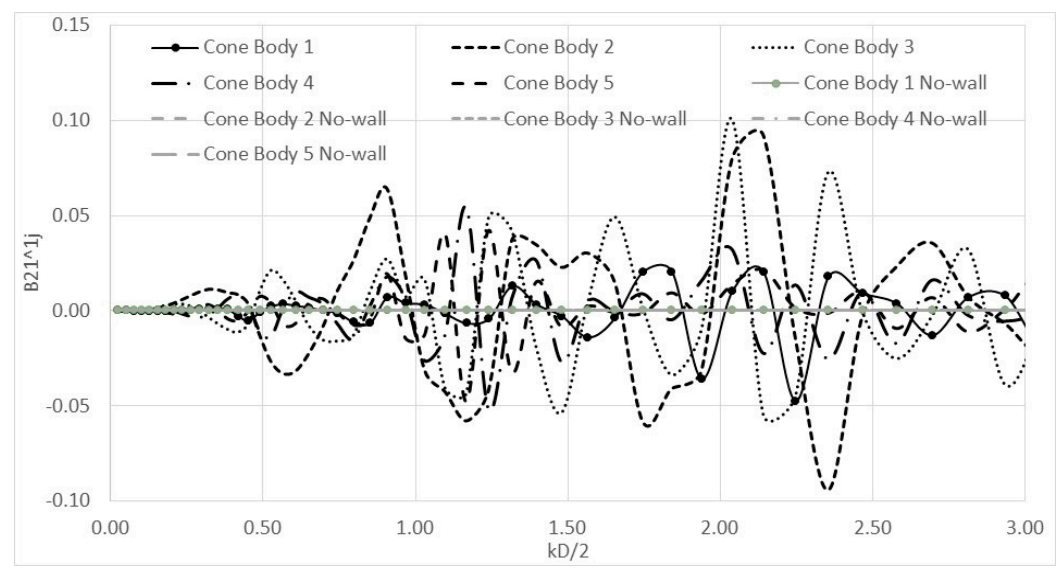

(b)

Figure 23. Dimensionless added mass (a) and damping coefficient (b) of the 1st conical floater in the sway direction due to the forced motion in surge of the $j$-th conical floater $(j=1,2, \ldots, 5)$ against $\mathrm{kD} / 2$, for the parallel arrangement, $C_{1}$. 
Next, indicative results concerning the absorbed wave power by the three examined arrangements (i.e., parallel, rectangular and perpendicular to the wall) for the case of the cylindrical floater-array are given. The cylindrical floaters of the array are assumed to oscillate at the heave direction, as heaving WECs. The power absorbed by each floater equals to [50]:

$$
p_{q}=0.5 b_{P T O} \omega^{2}\left|x_{30}^{q}\right|^{2}
$$

Here, $x_{30}^{q}$ is the complex amplitude of $q$ floater's motion in the vertical direction obtained from the solution of the linear system of motion equations (see Appendix D); $\omega$ is the wave frequency and $b_{\text {PTO }}$ represents the damping coefficient that originates from the Power Take Off (PTO) mechanism, modeled as a linear damping system actuated from the heave motion of the floater. In the present analysis all the examined cylindrical floaters are considered to have the same PTO characteristics, which for the sake of presenting some initial numerical results are assumed, according to [50], equal to the heave radiation damping of the isolated cylindrical floater at its heave natural frequency. Considering the draught and the radius of the floaters equal to $5 \mathrm{~m}$ and the mass of each floater equals to $402.5 \mathrm{t}$, the heave natural frequency of the floater equals to $1.088 \mathrm{rad} / \mathrm{s}$, leading to $b_{P T O}=73.88 \mathrm{kNm} / \mathrm{s}$.

In the Figure 24 the absorbed power by the first cylindrical floater (see Figure 6) of each examined arrangement is presented against the corresponding results of the same array arrangements without the presence of the breakwater. It can be observed that the wall influences the absorbed wave power by the floater. More specifically, near the natural frequency of the floater (i.e., $\mathrm{kD} / 2=0.68$ ) the presence of the wall causes a significant increase of the floater's absorbed power value compared to its no wall counterpart. This is in line with the expected influence of the vertical wall, which actually offers to the WEC the reflected wave for harvesting. This influence is evident in the low frequency regime of e.g., Figure 9 (wave frequencies up to approximately $0.25 \mathrm{rad} / \mathrm{s}$ ), where the vertical exciting wave forces on the first floater of the examined array configuration are depicted. However, for higher than $0.25 \mathrm{rad} / \mathrm{s}$ wave frequencies the wall's influence is affected by the hydrodynamic interactions between the floaters and the wall, depending on the specific geometrical arrangement and the distances $l_{w}$. These interactions can minimize the excitation forces and reduce the absorbed power. Moreover, it has to be stressed that the wave power absorption by a floater in front of a wall and its comparison with the no wall case is affected by the PTO damping coefficient which has to be properly selected to accommodate the importance of the hydrodynamic interference effects. It is therefore evident that the effect of the vertical wall on the WECs efficiency should be further examined for different PTO damping coefficients, distances between the wall and the floaters and various angles of wave propagation and in the entire range of the wave frequencies of interest, in order to depict accurately the influence of the wall to the WECs efficiency.

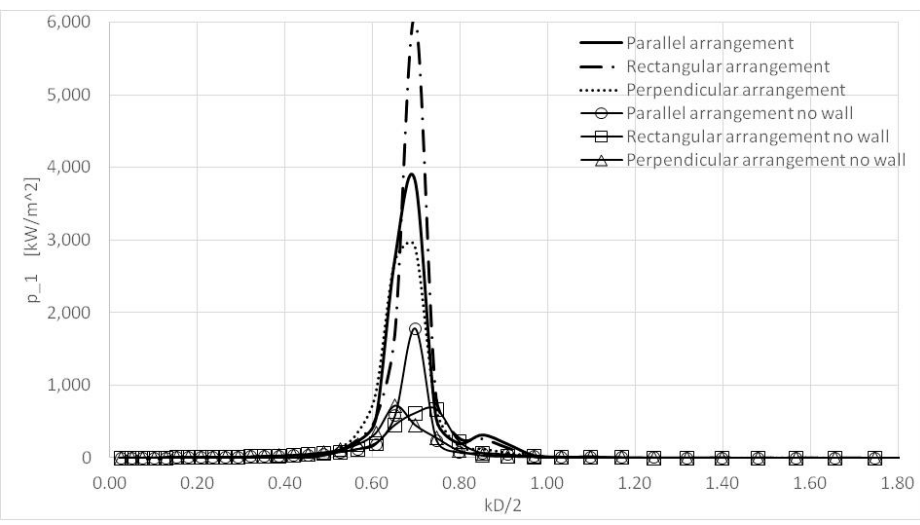

Figure 24. Absorbed wave power by the 1 st cylindrical floater against $\mathrm{kD} / 2$, for the parallel, $\mathrm{C}_{1}$, rectangular, $\mathrm{C}_{2}$; and perpendicular, $\mathrm{C}_{3}$ arrangement. The results are also compared with the absorbed power of the same floater of the arrangements without the presence of the wall. 


\section{Conclusions}

This study dealt with the determination of the hydrodynamic loads on an array of floaters in front of a vertical breakwater of infinite length. The image method has been applied to simulate the effect of the breakwater on the array and the multiple scattering approach has been used to evaluate the interaction phenomena between the WECs.

Three different types of floaters have been studied, i.e., a cylindrical; a conical and a semi-spherical floater, as well as three different types of array configurations in front of the vertical wall have been investigated, i.e., a parallel, a perpendicular and a rectangular array. Based on the theoretical computations shown and discussed in the dedicated sections, the main findings of the present research contribution concern the effect of the breakwater on the exciting wave forces and the hydrodynamic coefficients of the floaters, at every examined configuration, which should not be neglected when designing a WEC array in front of a vertical wall. This effect, the significance of which is depending on the distances between the floaters and the wall, causes an increase or decrease of the values of the exciting loads and the hydrodynamic coefficients of the floaters, at specific wave frequencies. On top of that, it is shown that the type of the floater dictates by a large expense the exciting wave loads and its hydrodynamic characteristics compared to the effect that the different examined array configuration may have on these hydrodynamic parameters. The presented figures showed that the cylindrical floater is characterized by higher values of surge exciting forces, as well as, surge hydrodynamic added mass and damping coefficients, at every array configuration, compared to those of the conical and semi-spherical floaters. However, this is not the case for the heave damping coefficients of the cylindrical floater, which attain lower values compared to the corresponding ones of the other examined floaters.

Concluding, the effect of the breakwater on the exciting wave loads and hydrodynamic coefficients of a floater, when the latter is a part of an array placed in front of the wall, increases or decreases in dependence to the ratio of the distance between the wall and the breakwater and the radius of the examined floater. However, neither the existence of the breakwater nor the arrangement of the array with respect to the incoming wave seem to have a greater influence on the values of the hydrodynamic forces and coefficients of a floater than its geometrical characteristics. Nevertheless, the present research will be continued further by determining in detail the power efficiency of the array and the q-factor [39] of the system, as well as their interaction with the presence of the breakwater, the shape of the floaters and their position in the array towards the incoming wave.

Author Contributions: Conceptualization, S.A.M. and G.M.K.; methodology, S.A.M., D.N.K., G.M.K.; software, S.A.M., D.N.K.; validation, D.N.K., G.M.K.; formal analysis, G.M.K.; investigation, D.N.K., G.M.K. and S.A.M.; data curation, D.N.K., G.M.K.; writing-original draft preparation, D.N.K.; writing-review and editing, D.N.K., S.A.M., G.M.K.; supervision, S.A.M., G.M.K.; project administration, S.A.M. All authors have read and agreed to the published version of the manuscript.

Funding: This research received no external funding.

Conflicts of Interest: The authors declare no conflict of interest.

\section{Nomenclature}

$N \quad$ Number of vertical axisymmetric floaters

d Water depth

$\omega \quad$ Wave frequency

A Wave amplitude

$\theta \quad$ Angle of wave propagation with respect to the positive $x$-axis

D Outer diameter of the examined floater

$h \quad$ Distance of the floater's bottom from the sea bed

$l_{\mathrm{W}} \quad$ Distance between the center of the closest to the wall floater and the breakwater

$l_{\mathrm{b}} \quad$ Distance between adjacent floaters 
$\varphi^{q} \quad$ Potential function describing the fluid flow around the $q$ floater, i.e.,

$\varphi^{q} \quad \Phi\left(r_{q}, \theta_{q}, z_{q} ; t\right)=\operatorname{Re}\left[\varphi^{q}\left(r_{q}, \theta_{q}, z_{q}\right)\right]$

$\varphi_{0} \quad$ Velocity potential of the undisturbed incident wave

$\varphi_{s}^{q} \quad$ Velocity potential of the scattered wave field around the $q$ floater

$\varphi_{j}^{q} \quad$ Velocity potential of the radiated wave field around the $q$ floater

$\varphi_{D}^{q} \quad$ Diffraction velocity potential around the $q$ floater

$J_{m} \quad$ The $m$-th order Bessel function of the first kind

$k \quad$ Wave number

$\left(l_{0 q}, \theta_{0 q}\right) \quad$ The polar coordinates of the $q$ floater center relative to the origin of the global co-ordinate system

$Z_{0}, Z_{n} \quad$ Orthonormal functions

$\dot{x}_{j 0}^{q} \quad$ The complex velocity amplitude of $q$ floater's motion in the $j$-th direction

I The infinite ring element around the $q$ floater

$I I I_{p} \quad$ The $p$-th ring element, $p=1, \ldots, L$, below the $q$ floater

$\alpha_{p} \quad$ The radius of the $p$-th ring element of type III

$h_{p} \quad$ Distance of the upper surface of the $p$-th ring element of type III from the sea bottom

$H_{m} \quad$ The $m$-th order Hankel function of first kind

$K_{m} \quad$ The $m$-th order modified Bessel function of second kind

$F_{k, m n}^{q}$

$F_{k, m n_{p}}^{q,} \quad$ The unknown Fourier coefficients

$F_{k, m n_{p}}^{* q}$

$\epsilon_{n_{p}} \quad$ The Neumann's symbol, $p=1, \ldots, L$

$a_{n} \quad$ The roots of the transcendental equation: $\omega^{2}+g a_{n} \tan \left(a_{n} d\right)=0$

$\rho \quad$ Sea water density

$F_{D, i}^{q} \quad$ The exciting wave forces on the $q$ floater in the $i$-th direction

$F_{i j}^{q p}$

$S_{q}$

The hydrodynamic reaction forces acting on the $q$ floater in the $i$-th direction, due to the forced oscillation of the $p$ floater in the $j$-th direction

$S_{q} \quad$ The mean wetted surface of the $q$ floater

$\mathrm{n}_{i} \quad$ The generalized normal components

$r$ The position vector of a point on the wetted surface $S_{q}$ with respect to the reference co-ordinate

$r \quad$ system of $q$ floater

$a_{i, j}^{q, p} \quad$ The added mass of the $q$ floater in $i$-th direction due to the forced oscillation of the $p$ floater in the

j-th direction

$\beta_{i, j}^{q, p} \quad$ The damping coefficient of the $q$ floater in $i$-th direction due to the forced oscillation of the $p$ floater

in the $j$-th direction

$\mathrm{b}_{\mathrm{PTO}} \quad$ The PTO damping coefficient

$p_{q} \quad$ Absorbed wave power by the $q$ floater

\section{Appendix A}

In the Tables A1-A3 the hydrodynamic characteristics in heave of the 3rd floater of an array of five floaters in front of a vertical breakwater (arrangement $C_{3}$, Figure 6) due to the heave motion of the $p$-th body in the array $(p=1, \ldots, 5)$, i.e., $A_{333 p}=\frac{a_{3,3}^{3, p}}{\frac{\rho \mathrm{D}^{3}}{8}} ; B_{333 p}=\frac{\beta_{3,3}^{3, p}}{\frac{u \mathrm{D}^{3}}{8}}$, (see Equation (19)), are presented against $\mathrm{kD} / 2$, for the examined arrays of conical, semi-spherical and cylindrical floaters, respectively. 
Table A1. Hydrodynamic characteristics of the 3rd floater of the array of five conical floaters.

\begin{tabular}{|c|c|c|c|c|c|c|c|c|c|c|}
\hline $\mathrm{kD} / 2$ & $33^{\wedge} 31$ & B33^31 & A33^32 & B33^32 & A33^33 & B33^33 & A33^34 & В33^34 & A33^35 & B33^35 \\
\hline 0.0760 & 2360 & 1028 & 3 & 1.2179 & 2.4640 & 0.89 & 0 & 0.3629 & 0.21 & 6327 \\
\hline 0.1805 & 0.0252 & .7306 & -0.3496 & -0.6498 & & 5191 & & -0.5031 & -0.7096 & 6787 \\
\hline 0.2928 & -0.7786 & -0.0826 & 0.2841 & 0.0017 & .0292 & 0917 & & -0.5122 & -0.6660 & .2569 \\
\hline 0.3536 & (2709 & -0.2549 & 0.0789 & 0.0752 & & & & & & -0.2889 \\
\hline 0.4894 & & -0.4 & 0. & & & & & & & 3393 \\
\hline 0.5665 & & -0 . & -0.2 & 0.1 & & & & & & 010 \\
\hline 15 & & -0 & -0 . & 0.2 & & & & 27 & & 111 \\
\hline 0.7459 & & -0 . & 0.2 & -0.1 & 1.1 & 22 & & -0 & F & 1059 \\
\hline 0.8511 & & & & 0.2 & 1.0 & & & & & 0.0828 \\
\hline 0.9683 & 0.1613 & 0.1726 & -0.1883 & -0.0104 & 1.1807 & 0.8200 & -0 & 0.0 & 0.0755 & .2864 \\
\hline 1.0984 & -0.1636 & 0.1469 & 0.0456 & -0.0476 & 1.0931 & .7917 & -0 . & -0.1468 & -0.1 & 0.1967 \\
\hline 1.3980 & -0.0915 & -0.1084 & -0.0460 & 0.1049 & 0.9388 & 0.6009 & -0.0733 & 0.0654 & -0.1622 & -0.1120 \\
\hline 1.5668 & 0.1309 & -0.0473 & 0.1312 & 0.0015 & & 0.6763 & & 0.0004 & & -0.0474 \\
\hline 1.7476 & & -0.0753 & -0.0075 & 0.0715 & & .5286 & & 0.0632 & 0.1197 & -0.0466 \\
\hline 1.9397 & & & & & & & & -0.0302 & & 0.0677 \\
\hline & & & & & & & & & & 0.0764 \\
\hline & & & & & & & & & & -0.0015 \\
\hline 2.8148 & 0.0482 & -0.0051 & -0.0109 & -0.0057 & 0.8368 & 0.2777 & -0.0160 & -0.0170 & 0.0489 & 0.0003 \\
\hline
\end{tabular}

Table A2. Hydrodynamic characteristics of the 3rd floater of the array of five semi-spherical floaters.

\begin{tabular}{|c|c|c|c|c|c|c|c|c|c|c|}
\hline kD/2. & A33^31 & B33^31 & A33^32 & B33^32 & A33^33 & B33^33 & A33^34 & B33^34 & A33^35 & B33^35 \\
\hline 0.0760 & -0.8635 & 1.2148 & -0.2368 & 1.1000 & 2.3526 & 0.8922 & 0.2178 & 0.6306 & -0.1497 & 0.3611 \\
\hline 0.1805 & -0.3377 & -0.6459 & -0.0255 & 0.7255 & 2.0528 & 1.5033 & -0.7073 & 0.6624 & -0.5302 & -0.5045 \\
\hline 0.2928 & 0.2713 & 0.0112 & -0.7435 & -0.0973 & 1.8795 & 1.0634 & -0.6481 & 0.2397 & 0.1851 & -0.4980 \\
\hline 0.3536 & 0.0697 & 0.0699 & -0.2516 & -0.2337 & 1.3792 & 1.1262 & -0.3762 & -0.2743 & 0.3186 & 0.1590 \\
\hline 0.4894 & 0.1053 & 0.1708 & -0.3529 & -0.4431 & 1.4818 & 1.0611 & -0.1367 & -0.5065 & -0.2121 & 0.2974 \\
\hline 0.5665 & -0.2086 & 0.1730 & 0.2169 & -0.3894 & 1.1993 & 0.7768 & -0.0269 & -0.2731 & -0.1417 & 0.0625 \\
\hline 0.6515 & -0.1809 & -0.2476 & 0.1228 & -0.1022 & 1.1718 & 0.9735 & 0.3082 & -0.1472 & 0.0078 & -0.2533 \\
\hline 0.7459 & 0.1729 & -0.0814 & 0.2182 & -0.0751 & 0.9993 & 0.6822 & 0.1856 & -0.0862 & 0.1099 & -0.1063 \\
\hline 0.8511 & 0.1182 & 0.2058 & 0.1870 & 0.1343 & 0.9866 & 0.6190 & 0.1473 & 0.0605 & 0.0313 & 0.0802 \\
\hline 0.9683 & -0.1454 & -0.0338 & 0.1254 & 0.1400 & 1.0568 & 0.6282 & 0.0262 & 0.2212 & -0.1289 & 0.0170 \\
\hline 1.0984 & 0.0373 & -0.0214 & -0.1192 & 0.0709 & 0.9910 & 0.5761 & -0.0992 & 0.1234 & 0.0071 & -0.1188 \\
\hline 1.3980 & -0.0485 & 0.0664 & -0.0432 & -0.0657 & 0.9364 & 0.3272 & -0.0918 & -0.0885 & -0.0619 & 0.0327 \\
\hline 1.5668 & 0.1177 & -0.0224 & 0.1128 & -0.0457 & 1.0162 & 0.3594 & 0.1023 & -0.0503 & 0.0942 & -0.0324 \\
\hline 1.7476 & -0.0175 & 0.0401 & 0.0557 & -0.0414 & 0.9123 & 0.2609 & 0.0660 & -0.0130 & -0.0058 & 0.0377 \\
\hline 1.9397 & -0.0006 & -0.0068 & 0.0105 & 0.0251 & 0.9558 & 0.2206 & 0.0105 & 0.0300 & -0.0018 & -0.0174 \\
\hline 2.1428 & 0.0254 & 0.0013 & -0.0227 & 0.0273 & 0.9354 & 0.2011 & -0.0070 & 0.0384 & 0.0186 & 0.0096 \\
\hline 2.3564 & 0.1268 & 0.0071 & 0.1106 & 0.0097 & 1.0873 & 0.1657 & 0.0914 & 0.0234 & 0.0862 & 0.0364 \\
\hline 2.8148 & -0.0019 & -0.0036 & 0.0110 & 0.0014 & 0.9984 & 0.0946 & 0.0089 & -0.0001 & -0.0010 & -0.0021 \\
\hline
\end{tabular}

Table A3. Hydrodynamic characteristics of the 3rd floater of the array of five cylindrical floaters.

\begin{tabular}{|c|c|c|c|c|c|c|c|c|c|c|}
\hline $\mathrm{kD} / 2$ & A33^31 & B33^31 & A33^32 & B33^32 & A33^33 & B33^33 & A33^34 & B33^34 & A33^35 & B33^35 \\
\hline 0.0760 & 0.8664 & 2090 & 2374 & 0945 & 0262 & 8876 & .220 & 6269 & -0.1477 & 0.3583 \\
\hline & & & & & & & & & & 5018 \\
\hline 0.2928 & 84 & 224 & & -0.1127 & 78 & & & & 14 & 4677 \\
\hline 0.3536 & 0.0568 & 00609 & 2102 & -0.2000 & 2.0120 & 68 & 84 & 468 & 0.2732 & .1608 \\
\hline 0.4894 & 0.0873 & 54 & -0.2938 & -0.3956 & 21036 & 971 & -0.0964 & -0 . & -0.2007 & 0.2357 \\
\hline & & & & & & & & & & 0.0530 \\
\hline 0.6515 & -0.1 & 072 & & -0 . & & & & & & -0.1837 \\
\hline 0.7459 & & & & & & & & & & 606 \\
\hline & & & & & & & & & & \\
\hline & & & & & & & & & & \\
\hline & & & - & & & & & & & 510 \\
\hline & $-c$ & & & & & & & & -0 & 0.0041 \\
\hline 1.5668 & 0. & -0. & 0.0 & -0. & 1.9 & 34 & & -0 & 0.0 & -0.0210 \\
\hline 1.7476 & -0.0050 & 0.0059 & 0.01 & -0.0063 & 1.90 & 0.0426 & & & -0 . & 0.0057 \\
\hline 1.9397 & 0.0000 & -0.0007 & 0.0013 & 0.0024 & 1.9347 & 0.0278 & 0.0065 & & 0.0003 & -0.0019 \\
\hline 2.1428 & (2007 & 0.0009 & -0.0018 & 0.0020 & 1.95 & 0.0177 & -0.0010 & & 0.0018 & 0.0017 \\
\hline 2.3564 & 0.0176 & 0.0108 & 0.0168 & 0.0110 & 1.9931 & 0.0212 & 0.0126 & 0.0118 & 0.0125 & 0.0120 \\
\hline 2.8148 & 0.0001 & -0.0002 & 0.0007 & 0.0001 & 2.0082 & 0.0032 & 0.0010 & -0.0003 & 0.0002 & 0.0003 \\
\hline
\end{tabular}




\section{Appendix B}

In the Table A4 the hydrodynamic characteristics in sway of the 1st cylindrical floater of an array of five cylindrical floaters in front of a vertical breakwater (arrangement $C_{1}$, Figure 6) due to the sway motion of the $p$-th body in the array $(p=1, \ldots, 5)$, i.e., $A_{221 p}=\frac{a_{2,2}^{1, p}}{\frac{\rho \mathrm{D}^{3}}{8}} ; B_{221 p}=\frac{\beta_{2,2}^{1, p}}{\frac{\omega \rho \mathrm{D}^{3}}{8}}$, (see Equation (19)), are presented against kD/2.

Table A4. Hydrodynamic characteristics of the 1st floater of the array of five cylindrical floaters.

\begin{tabular}{ccccccccccc}
\hline $\mathbf{k D} / \mathbf{2}$ & $\mathbf{A 2 2} \mathbf{1 1}$ & $\mathbf{B 2 2} \mathbf{1 1}$ & $\mathbf{A 2 2} \mathbf{1 2}$ & $\mathbf{B 2 2} \mathbf{1 2}$ & $\mathbf{A 2 2} \mathbf{1 3}$ & $\mathbf{B 2 2} \mathbf{1 3}$ & $\mathbf{A 2 2} \mathbf{1 4}$ & $\mathbf{B 2 2} \mathbf{1 4}$ & $\mathbf{A 2 2} \mathbf{1 5}$ & $\mathbf{B 2 2}^{\wedge} \mathbf{1 5}$ \\
\hline 0.0760 & 2.0240 & 0.0183 & -0.0333 & 0.0156 & -0.0229 & 0.0089 & -0.0208 & 0.0000 & -0.0165 & -0.0084 \\
0.1805 & 2.0946 & 0.0988 & -0.1033 & 0.0271 & -0.0532 & -0.0743 & 0.0491 & -0.0597 & 0.0608 & 0.0351 \\
0.2928 & 2.1574 & 0.2369 & -0.1880 & -0.1245 & 0.1852 & -0.0818 & -0.0532 & 0.1774 & -0.0791 & -0.1307 \\
0.3536 & 2.2015 & 0.3541 & -0.1477 & -0.3186 & 0.2236 & 0.2107 & -0.2359 & -0.0907 & 0.2053 & -0.0131 \\
0.4894 & 2.3223 & 0.4508 & 0.2054 & -0.3321 & -0.3508 & 0.0840 & 0.3204 & 0.1705 & -0.1637 & -0.3285 \\
0.5665 & 2.3637 & 0.6022 & 0.4733 & -0.0971 & -0.2355 & -0.5107 & -0.3272 & 0.3323 & 0.3707 & 0.2725 \\
0.6515 & 2.4707 & 0.8085 & 0.5054 & 0.3262 & 0.5083 & -0.3324 & -0.1108 & -0.6708 & -0.5270 & -0.2038 \\
0.7459 & 2.3836 & 1.4354 & 0.0344 & 0.9222 & 0.1344 & 0.9514 & 0.3748 & 0.6947 & 0.4932 & 0.3823 \\
0.8511 & 2.5715 & 1.3667 & -0.0572 & 0.4063 & -0.6186 & 0.0419 & -0.7277 & -0.2513 & -0.5758 & -0.4337 \\
0.9683 & 1.8682 & 1.6947 & -0.4459 & 0.1238 & 0.3490 & -0.6673 & 0.6573 & 0.1634 & -0.0601 & 0.6370 \\
1.0984 & 1.8146 & 1.6999 & -0.8365 & -0.2386 & 0.7164 & 0.4411 & -0.7716 & -0.0823 & 0.6550 & -0.3169 \\
1.3980 & 0.9078 & 1.3463 & 0.5921 & -0.4201 & 0.3349 & -0.0537 & -0.0863 & -0.2994 & -0.4034 & 0.0289 \\
1.5668 & 0.9926 & 1.4841 & 0.5586 & 0.7083 & -0.2482 & 0.4221 & -0.1401 & 0.4517 & -0.2159 & 0.3162 \\
1.7476 & 0.2887 & 1.3876 & -0.4381 & 0.5763 & 0.4545 & -0.0133 & 0.2373 & 0.1227 & -0.1345 & 0.1795 \\
1.9397 & 0.7768 & 1.0233 & -0.5525 & -0.3183 & 0.0166 & 0.0321 & -0.1709 & -0.1282 & 0.0572 & 0.1157 \\
2.1428 & 0.3299 & 1.0565 & 0.2992 & -0.5280 & 0.0286 & 0.3425 & -0.3123 & -0.1373 & 0.1857 & 0.1297 \\
2.3564 & 0.4857 & 0.8862 & 0.3346 & 0.1012 & 0.1713 & -0.1660 & -0.0121 & 0.1464 & 0.0771 & 0.1877 \\
2.8148 & 0.4188 & 0.6489 & -0.1544 & 0.0188 & 0.2062 & 0.1173 & 0.0848 & -0.0616 & -0.1132 & -0.0147 \\
\hline
\end{tabular}

\section{Appendix C}

In the Table $\mathrm{A} 5$ the hydrodynamic characteristics in sway of the 1st conical floater of an array of five conical floaters in front of a vertical breakwater (arrangement $C_{1}$, Figure 6) due to the surge motion of the $p$-th body in the array $(p=1, \ldots, 5)$, i.e., $A_{211 p}=\frac{a_{2,1}^{1, p}}{\frac{\rho \mathrm{D}^{3}}{8}} ; B_{211} 1 p=\frac{\beta_{2,1}^{1, p}}{\frac{\omega \rho \mathrm{D}^{3}}{8}}$, (see Equation (19)), are presented against $\mathrm{kD} / 2$.

Table A5. Hydrodynamic characteristics of the 1st floater of the array of five conical floaters.

\begin{tabular}{ccccccccccc}
\hline $\mathbf{k D} / \mathbf{2}$ & $\mathbf{A 2 1} \mathbf{1 1}$ & $\mathbf{B 2 1} \mathbf{1 1}$ & $\mathbf{A 2 1} \mathbf{1 2}$ & $\mathbf{B 2 1} \mathbf{1 2}$ & $\mathbf{A 2 1} \mathbf{1 3}$ & $\mathbf{B 2 1} \mathbf{1 3}$ & $\mathbf{A 2 1} \mathbf{1 4}$ & $\mathbf{B 2 1} \mathbf{1 4}^{\mathbf{A}}$ & $\mathbf{A 2 1} \mathbf{1 5}$ & $\mathbf{B 2 1} \mathbf{1 5}$ \\
\hline 0.0760 & 0.0000 & 0.0000 & 0.0025 & 0.0001 & 0.0010 & 0.0002 & 0.0005 & 0.0002 & 0.0002 & 0.0002 \\
0.1805 & 0.0002 & 0.0000 & 0.0044 & 0.0024 & 0.0006 & 0.0027 & -0.0014 & 0.0010 & -0.0010 & -0.0006 \\
0.2928 & 0.0000 & 0.0006 & 0.0008 & 0.0099 & -0.0062 & -0.0002 & 0.0014 & -0.0034 & 0.0018 & 0.0024 \\
0.3536 & -0.0007 & 0.0011 & -0.0069 & 0.0110 & -0.0043 & -0.0070 & 0.0056 & 0.0015 & -0.0043 & 0.0006 \\
0.4894 & 0.0050 & -0.0008 & -0.0276 & -0.0125 & 0.0193 & 0.0097 & -0.0099 & -0.0104 & 0.0026 & 0.0077 \\
0.5665 & 0.0010 & 0.0035 & -0.0034 & -0.0329 & -0.0107 & 0.0162 & 0.0137 & 0.0009 & -0.0058 & -0.0071 \\
0.6515 & -0.0020 & 0.0016 & 0.0269 & -0.0224 & -0.0163 & -0.0079 & -0.0038 & 0.0072 & 0.0067 & 0.0043 \\
0.7459 & -0.0031 & -0.0013 & 0.0381 & 0.0108 & 0.0037 & -0.0160 & -0.0105 & -0.0070 & -0.0065 & -0.0007 \\
0.8511 & 0.0088 & -0.0066 & 0.0238 & 0.0484 & 0.0299 & 0.0095 & 0.0211 & 0.0023 & 0.0126 & -0.0044 \\
0.9683 & 0.0025 & 0.0040 & -0.0653 & 0.0189 & -0.0095 & 0.0073 & -0.0134 & 0.0066 & -0.0223 & -0.0142 \\
1.0984 & -0.0096 & -0.0015 & -0.0223 & -0.0425 & -0.0325 & -0.0407 & 0.0520 & -0.0127 & -0.0220 & 0.0408 \\
1.3980 & -0.0043 & 0.0033 & 0.0048 & 0.0346 & -0.0497 & -0.0195 & -0.0134 & 0.0265 & 0.0310 & 0.0155 \\
1.5668 & -0.0041 & -0.0140 & -0.0197 & 0.0303 & 0.0459 & 0.0043 & 0.0232 & 0.0050 & 0.0199 & 0.0026 \\
1.7476 & 0.0069 & 0.0205 & -0.0447 & -0.0583 & -0.0428 & 0.0014 & 0.0092 & 0.0090 & 0.0054 & -0.0011 \\
1.9397 & -0.0132 & -0.0358 & 0.0521 & -0.0315 & 0.0380 & -0.0102 & -0.0003 & 0.0157 & -0.0074 & -0.0014 \\
2.1428 & -0.0384 & 0.0204 & -0.0797 & 0.0922 & -0.0619 & -0.0546 & 0.0604 & -0.0223 & 0.0050 & 0.0217 \\
2.3564 & 0.0235 & 0.0179 & -0.0055 & -0.0941 & 0.0119 & 0.0728 & 0.0453 & -0.0255 & -0.0161 & -0.0010 \\
2.8148 & -0.0074 & 0.0071 & -0.0370 & 0.0074 & -0.0170 & 0.0323 & 0.0005 & -0.0024 & 0.0123 & -0.0109 \\
\hline
\end{tabular}

\section{Appendix D}

The equilibrium of the forces acting on the each floater of the array leads to the following system of differential equations of motion in the frequency domain, $(q=1,2, \ldots, N)$, i.e.,: 


$$
\sum_{p=1}^{N} \sum_{j=1}^{6}\left(\delta_{p, q} m_{k j}^{q}+a_{k, j}^{q, p}\right) \ddot{x}_{j 0}^{p}+\left(\beta_{k, j}^{q, p}+\delta_{p, q} \delta_{j, 3} b_{P T O}^{p}\right) \dot{x}_{j 0}^{p}+\delta_{p, q} c_{k j}^{q} x_{j 0}^{p}=F_{D, k}^{q}, k=1, \ldots, 6
$$

where $x_{j 0}^{p}$ is the 6-degree displacement vector of the $p$ floater of the array; $m_{k j}^{q}$ is the mass matrix of the $q$ floater; $a_{k, j}^{q, p}$ is the frequency-dependent hydrodynamic mass matrix and $\beta_{k, j}^{q, p}$ is the frequency-dependent damping matrix of the $q$ floater in the $k$ th direction due to the forced oscillation of the $p$ floater in the $j$ th direction (see Equation (19)); $c_{k j}^{q}$ is the stiffness matrix; $F_{D, k}^{q}$ represents the exciting force on the $q$ floater in the $k$ th direction (see Equation (17)); $b_{P T O}^{p}$ is the damping coefficients that originate from the PTO mechanism and $\delta_{p, q}, \delta_{j, 3}$ are Kronecker delta.

\section{References}

1. McCormick, M.E. Ocean Wave Energy Conversion; Courier Corporation: North Chelmsford, MA, USA, 1981.

2. Pelc, R.; Fujita, R.M. Renewable energy from the ocean. Mar. Policy 2002, 26, 471-479. [CrossRef]

3. Falnes, J. A review of wave-energy extraction. Mar. Struct. 2007, 20, 185-201. [CrossRef]

4. Falcao, A.F.O. Wave energy utilization: A review of the technologies. Renew. Sustain. Energy Rev. 2010, 14, 899-918. [CrossRef]

5. Aderinto, T.; Li, H. Ocean wave energy converters: Status and Challenges. Energies 2008, 11, 1250. [CrossRef]

6. Giebhardt, J.; Kracht, P.; Dick, C.; Salcedo, F. Report on Grid Integration and Power Quality Testing; Deliverable 4.3 Final; Marinet: Singapore, 2014.

7. Magagna, D.; Uihlein, A. Ocean energy development in Europe: Current status and future perspectives. Int. J. Mar. Energy 2015, 11, 84-104. [CrossRef]

8. Mustapa, M.A.; Yaakob, O.B.; Ahmed, Y.M.M.; Rheem, C.K.; Koh, K.K.; Faizul, A.A. Wave energy device and breakwater integration: A review. Renew. Sustain. Energy Rev. 2017, 77, 43-58. [CrossRef]

9. Rusu, E. Evaluation of the wave energy conversion in various coastal environments. Energies 2014, 7, 4002-4018. [CrossRef]

10. Cascajo, R.; Garcia, E.; Quiles, E.; Correcher, A.; Morant, F. Integration of marine wave energy convertres into seaports: A case study in port of Valencia. Energies 2019, 12, 787. [CrossRef]

11. Mavrakos, S.A.; Katsaounis, G.M.; Nielsen, K.; Lemonis, G. Numerical performance investigation of an array of heaving wave power converters in front of a vertical breakwater. In Proceedings of the 14th International Offshore and Polar Engineering Conference (ISOPE 2004), Toulon, France, 23-28 May 2004.

12. Mavrakos, S.A.; Katsaounis, G.M.; Kladas, A.; Kimoulakis, N. Numerical and experimental investigation of performance of heaving WECs coupled with DC generators. In Proceedings of the 9th European Wave and Tidal Energy Conference, Southampton, UK, 5-9 September 2011.

13. Teng, B.; Ning, D.Z. Wave diffraction from a uniform cylinder in front of a vertical wall. Ocean Eng. 2003, 21, $48-52$.

14. Teng, B.; Ning, D.Z.; Zhang, X.T. Wave radiation by a uniform cylinder in front of a vertical wall. Ocean Eng. 2004, 31, 201-224. [CrossRef]

15. Zheng, S.; Zhang, Y. Wave diffraction from a truncated cylinder in front of a vertical wall. Ocean Eng. 2015, 104, 329-343. [CrossRef]

16. Zheng, S.; Zhang, Y. Wave radiation from a truncated cylinder in front of a vertical wall. Ocean Eng. 2016, 111, 602-614. [CrossRef]

17. Schay, J.; Bhattacharjee, J.; Soares, C. Numerical modelling of a heaving point absorber in front of a vertical wall. In Proceedings of the 32nd International Conference on Ocean, Offshore and Artic Engineering (OMAE 2013), Nantes, France, 9-14 June 2013. [CrossRef]

18. He, F.; Huang, Z. Using an oscillating water column structure to reduce wave reflection from vertical wall. J. Waterw. Port Coast. Ocean Eng. 2016, 142. [CrossRef]

19. Martins-rivas, H.; Mei, C.C. Wave power extraction from an oscillating water column at the tip of a breakwater. J. Fluid Mech. 2009, 626, 395-414. [CrossRef]

20. Martins-rivas, H.; Mei, C.C. Wave power extraction from an oscillating water column along a straight coast. Ocean Eng. 2009, 36, 426-433. [CrossRef]

21. Howe, D.; Nader, J.R. OWC WEC integrated within a breakwater versus isolated: Experimental and numerical theoretical study. Mar. Energy 2017, 20, 165-182. [CrossRef] 
22. Naty, S.; Viviano, A.; Foti, E. Wave energy exploitation system integrated in the coastal structure of a Mediterranean port. Sustainability 2016, 8, 1342. [CrossRef]

23. Naty, S.; Viviano, A.; Foti, E. Feasibility study of a WEC integrated in the port of Giardini Naxos, Italy. In Proceedings of the 35th Conference on Coastal Engineering, Antalya, Turkey, 17-20 November 2016.

24. He, F.; Huang, Z.; Law, A.W.K. Hydrodynamic performance of a rectangular floating breakwater with and without pneumatic chambers: An experimental study. Ocean Eng. 2012, 51, 16-27. [CrossRef]

25. Zheng, X.; Zeng, Q.; Liu, Z. Hydrodynamic performance of rectangular heaving buoys for an integrated floating breakwater. J. Mar. Sci. Eng. 2019, 7, 239. [CrossRef]

26. He, F.; Zhang, H.; Zhao, J.; Zheng, S.; Iglesias, G. Hydrodynamic performance of a pile-supported OWC breakwater: An analytical study. Appl. Ocean Res. 2019, 88, 326-340. [CrossRef]

27. Zhao, X.L.; Ning, D.Z.; Zhang, C.W.; Liu, Y.Y.; Kang, H.G. Analytical study on an oscillating buoy wave energy converter integrated into a fixed box-type breakwater. Math. Probl. Eng. 2017, 2017, 1-9. [CrossRef]

28. Zhao, X.L.; Ning, D.Z.; Liang, D.F. Experimental investigation on hydrodynamic performance of a breakwater-integrated WEC system. Ocean Eng. 2019, 171, 25-32. [CrossRef]

29. Salter, S.; Taylor, J.; Caldwell, N. Power conversion mechanisms for wave energy. J. Eng. Marit. Environ. 2002, 216, 1-27. [CrossRef]

30. Falnes, J.; Hals, J. Heaving buoys, point absorbers and arrays. Philos. Trans. R. Soc. A 2012, $370,246$. [CrossRef] [PubMed]

31. Rahmati, M.T.; Aggidis, G.A. Numerical and experimental analysis of the power output of a point absorber wave energy converter in irregular waves. Ocean Eng. 2016, 111, 483-492. [CrossRef]

32. Tampier, G.; Grueter, L. Hydrodynamic analysis of a heaving wave energy converter. Int. J. Mar. Energy 2017, 19, 304-318. [CrossRef]

33. Son, D.; Yeung, R. Real time implementation and validation of optimal damping control for a permanent magnet linear generator in wave energy extraction. Appl. Energy 2017, 208, 571-579. [CrossRef]

34. Gaspar, J.; Calvario, M.; Kamarlouei, M.; Soares, C. Power take-off concept for wave energy converters based on oil-hydraulic transformer units. Renew. Energy 2016, 86, 1232-1246. [CrossRef]

35. Spring, B.W.; Monkmeyer, P.L. Interaction of plane waves with a row of cylinders. In Proceedings of the 3rd Specialty Conference of Civil Engineering in Oceans ASCE, Newark, DE, USA, 9-12 June 1975; pp. 979-998.

36. Yeung, R.W.; Sphaier, S.H. Wave-interference effects on a truncated cylinder in a channel. J. Eng. Math. 1989, 23, 95-117. [CrossRef]

37. Yeung, R.W.; Sphaier, S.H. Wave-interference effects on a floating body in a towing tank. In Proceedings of the 4th International Symposium on Practical Design of Ships and Mobile Units (PRADS), Varna, Bulgaria, 23-28 October 1989.

38. Mavrakos, S.A. The scattered wave field by vertical cylinders in a narrow tank. In Proceedings of the 4 th National Symposium on Theoretical and Applied Mechanics, Xanthi, Greece, 26-29 June 1995; Volume II, pp. 819-829.

39. Twersky, V. Multiple scattering of radiation by an arbitrary configuration of parallel cylinders. J. Acoust. Soc. Am. 1952, 24, 42. [CrossRef]

40. Okhusu, M. Hydrodynamic forces on multiple cylinders in waves. In Proceedings of the International Symposium on the Dynamics of Marine Vehicles and Structures in Waves, London, UK, 1-5 April 1974.

41. Mavrakos, S.A.; Koumoutsakos, P. Hydrodynamic interaction among vertical axisymmetric bodies restrained in waves. Appl. Ocean Res. 1987, 9, 128-140. [CrossRef]

42. Mavrakos, S.A. Hydrodynamic coefficients for groups of interacting vertical axisymmetric bodies. Ocean Eng. 1991, 18, 485-515. [CrossRef]

43. Mavrakos, S.A.; McIver, P. Comparison of methods for computing hydrodynamic characteristics of arrays of wave power devices. Appl. Ocean Res. 1997, 19, 283-291. [CrossRef]

44. Konispoliatis, D.N.; Mavrakos, S.A. Hydrodynamic analysis of an array of interacting free-floating oscillating water column devices. Ocean Eng. 2016, 111, 179-197. [CrossRef]

45. Kokkinowrachos, K.; Mavrakos, S.; Asorakos, S. Behavior of vertical bodies of revolution in waves. Ocean Eng. 1986, 13, 505-538. [CrossRef]

46. Newman, J.N. Marine Hydrodynamics; MIT Press: Cambridge, UK, 1977.

47. Faltinsen, O.M. Sea Loads on Ships and Offshore Structures; Cambridge University Press: Cambridge, UK, 1990. 
48. Loukogeorgaki, E.; Chatjigeorgiou, I. Hydrodynamic performance of an array of wave energy converters in front of a vertical wall. In Proceedings of the 13th European Wave and Tidal Energy Conference (EWTEC), Naples, Italy, 1-6 September 2019.

49. Nader, J.R.; Zhu, S.P.; Cooper, P.; Steppenbelt, B. A finite element study of the efficiency of arrays of oscillating water column wave energy converters. Ocean Eng. 2012, 43, 72-81. [CrossRef]

50. Falnes, J. Ocean. Waves and Oscillating Systems: Linear Interactions Including Wave-Energy Extraction; Cambridge University Press: Cambridge, UK, 2002.

(C) 2020 by the authors. Licensee MDPI, Basel, Switzerland. This article is an open access article distributed under the terms and conditions of the Creative Commons Attribution (CC BY) license (http://creativecommons.org/licenses/by/4.0/). 